\title{
Article
}

\section{Emergence of standard-model-like fields and particles in gravitation-diffusion vacuum}

\author{
Vladimir A. Manasson 1,* \\ 1 Sierra Nevada Corporation, CA; v.a.manasson@cox.net \\ * Correspondence: v.a.manasson@cox.net; Tel.: +01-949-751-7606
}

\begin{abstract}
A number of experiments suggest that the elementary particles are non-local entities. A dissipative self-organization, that treats particles as open systems may provide a better understanding of the underlying phenomena than conservative models. The proposed toy model is such an attempt.

We found that self-gravitation (although accompanied by self-diffusion) may not only be compatible with the quantum phenomena but is perhaps the major reason for the existence of quantum fields. According to the proposed model, fields/particles (resemble those of the Standard Model) emerge from a dynamic self-organized medium (which we associate with vacuum dust) from competition between self-gravitation and self-diffusion. These forces produce turbulence in the form of vortices (which we call vacuum cells) that serve as the building blocks for fields and particles. Field/particle features and symmetries are based on their internal (intracellular) dynamics and vortex synchronization (intercellular dynamics).
\end{abstract}

This model allows for rough estimations of relative field coupling strengths, the quantity of charges and flavors, the probabilities of quark transmutations, the dimensionality and the topologies of phase spaces, and other Standard Model parameters that came not from the theory but rather determined by experiment.

Keywords: particle physics; unification of forces; self-organization; coupling constants; quark flavors; quark mixing amplitudes; quantum statistics; open systems; Feigenbaum universality; synchronization

\section{Introduction}

Our model is built on the premise that the Universe is essentially a dynamic open system that is far from thermodynamic equilibrium, and percolated by numerous energy flows. Our departure point is the hypothetical vacuum dust, which plays the role of an active medium that gives birth to fields/particles resembling those of the Standard Model (SM). Vacuum dust evolves under the influence of two omnipresent competing forces: self-gravitation and self-diffusion. These forces create antiparallel micro-flows. Under some conditions, these micro-flows lose tangential stability and produce vortices (which we call vacuum cells), a phenomenon observed in numerous active media and known as formation of Bénard cells [1].

A vacuum cell (vortex) is an open system, which exchanges dust particles with its neighbors and unorganized vacuum. The latter two represent the external forces that tend to destroy cells. Correspondingly, vacuum cell stability is the primary concern of this paper. Dynamically stable states emerge when self-gravitation and self-diffusion balance each other. External forces may shift the balance. However, after these perturbations subside, the vacuum cell asymptotically returns to its original state. Hence, we call cellular stability asymptotic, and dynamic-equilibrium states attractors. 
Asymptotic stability liberates vacuum cells from the custody of fixed boundaries, conservation principles, internal symmetries, and physical constants, which "protect" particles from disintegration "by laws" in the conservative frameworks.

In the proposed dissipative framework, time is irreversible and cannot mix with "reversible" space. They are separated from each other and represent a background without metrics. Local time and space metrics are rather attributes that are carried by vacuum cells. Global and local symmetries emerge when cells synchronize their dynamics. Relativistic time dilation emerges as an asymptotic stability phenomenon.

The erratic character of infinitely many external perturbations acting on open cells implies that a probabilistic framework is the only one possible. For the same reason, the model operates with collective phenomenological parameters, such as generic charge $\chi$, strength of feedback loops expressed as interchangeable parameters $A$ and $B$, the Lyapunov exponent $\lambda$ as a measure of asymptotic stability, energy $\mathcal{E}$, chemical potential $\mu$, and others.

To describe cellular evolutions, we use discrete-time (stroboscopic) iterated maps rather than differential equations. The approach allows us to account for complex phenomena like phase transitions (abrupt changes of phase dimensionality and topology) with simple one-dimensional map formalism. The downside of this simplification is our inability to describe the events between the stroboscopic instants $t_{n}$. However, this missing information is insignificant to the outcomes of the model. It is sufficient to assume that cellular trajectories converging toward their attractors are continuous and are sampled when they cross a selected Poincaré plane (Figure 1).

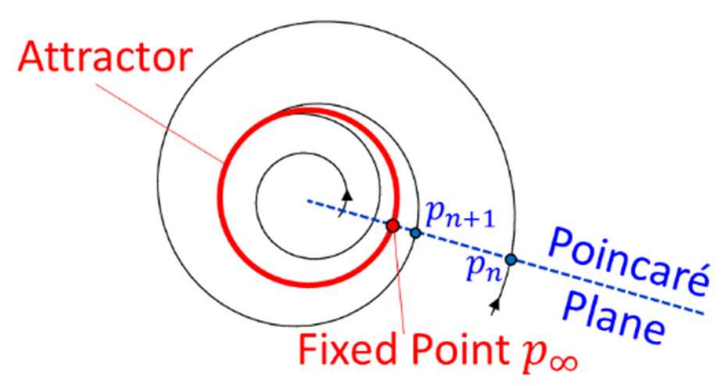

Figure 1. Discrete-time mapping of crossing points between cellular trajectories (black spirals) and Poincaré plane (shown in blue). The asymptotic state (attractor) is indicated by the red closed curve.

\section{Cellular Evolution}

We define $p$ as the probability that local vacuum dust at a given time $t$ effectively converges. Self-gravitation acts as a positive feedback: the bigger the $p$, the closer the dust particles come together and the stronger their self-attraction. Under positive feedback, $p$ increases with time (in the simplest case) as $p(t+\Delta t) \propto p(t) \Delta t$. Correspondingly, self-diffusion acts as a negative feedback: the closer the dust particles come together, the stronger their apparent repulsion. In the simplest case $p(t+\Delta t) \propto(1-p(t)) \Delta t$.

We combine both actions in a single discrete-time one-dimensional iterated map

$$
p_{n+1}=A p_{n}\left(1-p_{n}\right),
$$


where $\Delta t=\mathcal{T}=t_{n+1}-t_{n} ; \mathcal{T}$ is the cellular period; $p_{n+1}$ and $p_{n}$ are probabilities at time instants $t_{n+1}$ and $t_{n}$, and $A$ is the feedback amplification parameter. If $0 \leq A \leq 4, p_{n}$ are non-negative and smaller than 1.

The iterated map (1) describes vacuum dust evolution. It is known as a logistic map and is well characterized [2-4]. It represents a wide class of iterated maps with unimodal iterated functions and, like all of these maps, possess the Feigenbaum universality [4-7], which includes cascades of perioddoubling bifurcations and specific numerical relations to the fractal-type stability intervals (the Feigenbaum numbers). One of these relations, the Feigenbaum delta, $\delta_{F}=4.669 \ldots$, provides the relative width of intervals between bifurcations, and plays an important role in our simulations. The Feigenbaum universality downgrades the importance of the specific form of map (1) as any map with a unimodal iteration function (having a single extremum) would provide identical or close to identical results.

\section{Generic Charges. Antimatter}

We define a generic charge as

$$
\chi=2 p-1
$$

It inherits the probabilistic nature from probability $p$ and occupies the domain $|\chi| \leq 1$.

Unlike probabilities, the generic charge can be positive, negative, or equal to zero, and represent converging $\chi>0$, diverging $\chi<0$, or purely circular $\chi=0$ cellular flows (Figure 2).

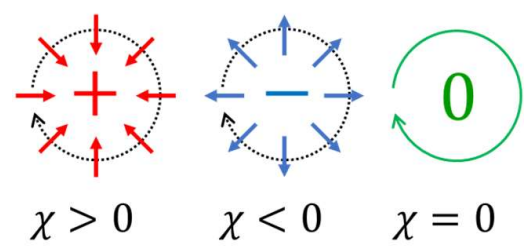

Figure 2. Charge polarity assignment (the directions of radial flows are opposite to the directions of the field lines adopted in electrodynamics).

For aesthetic reasons, we also substitute parameter $A$ with parameter $B:|B| \leq 1$

$$
B=\frac{A}{2}-1 .
$$

With substitutions (2) and (3), the original map takes the form

$$
\chi_{n+1}=\mathcal{L}\left(\chi_{n}, B\right)
$$

with iteration function

$$
\mathcal{L}(\chi, B)=B-(1+B) \chi^{2}
$$

Reversing all cellular radial microflows represents the operation of charge conjugation (transition from matter to anti-matter). In the proposed model, it is irrelevant for time inversion, which is a prohibited symmetry in dissipative systems (time inversion would convert asymptotic stability into chaos). Rather, it permutes self-gravitation and self-diffusion. The charge-conjugated map

$$
-\chi_{n+1}=\mathcal{L}\left(-\chi_{n}, B\right)=\mathcal{L}\left(\chi_{n}, B\right)
$$

describes the evolution of anti-cells, which are also field/particle building blocks in the model. 
Map (6) is a mirror copy of the map (4), where all charges are replaced with their conjugated copies. It has the same behavior as map (5), including stability, winding numbers, and positions of superattractors and bifurcation points in $B$-space. The combined evolution map is

$$
\chi_{n+1}= \pm \mathcal{L}\left(\chi_{n}, B\right)
$$

where plus and minus signs refer to cells and anti-cells respectively.

\section{Bifurcation Diagram. Cellular Phases. Circular Time Diagrams (CTD)}

A bifurcation diagram is a multivalued function $\chi_{\infty}(B)$ that depicts fixed points $\chi_{\infty}$ in $B$ space (Figure 3(a)).

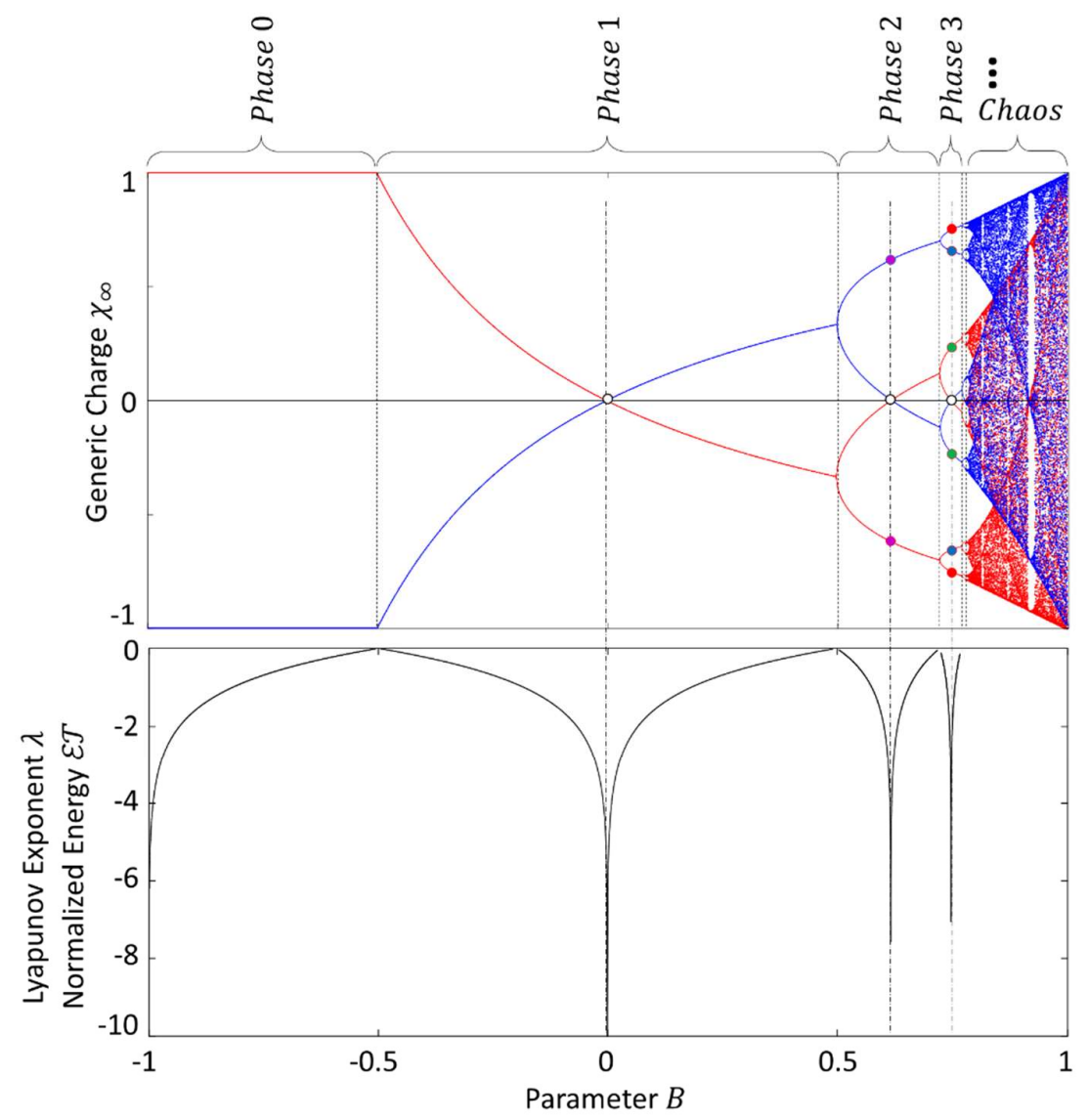

(a)

(b)

Figure 3. (a) Bifurcation diagram for cells (blue) and anti-cells (red); (b) Laypunov exponent $\lambda(B)$ and normalized energy $\mathcal{E}(B) \mathcal{T}$.

The cellular portion is shown in blue, and the charge-conjugated (anti-cellular) portion is shown in red. Each branch corresponds to one attractor loop. The number of branches reflect the attractor winding numbers $\mathcal{W}$ (Figure 4). At bifurcations, the number of branches double. Correspondingly, the number of attractor loops also doubles.
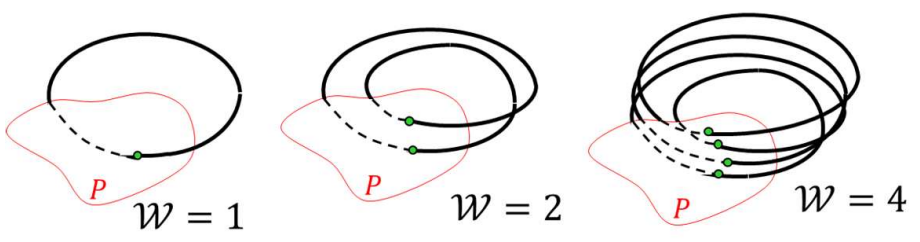
Figure 4. Attractors possessing one $(\mathcal{W}=1)$, two $(\mathcal{W}=2)$ and four $(\mathcal{W}=4)$ loops. $P$ is the Poincaré plane.

The abrupt changes in attractor topologies at bifurcations are followed by significant changes in cellular dynamics. This is reflected by dramatic changes of the iteration function, cellular phase space dimensionality, size and topology, the number of charges, asymptotic stability, chemical potentials, partition functions, restoring forces, etc. Therefore, we associate period-doubling bifurcations with cellular phase transitions. In this paper, we designate different phases (intervals between the bifurcations) by the letter $j, j=0,1,2,3 \ldots$. Superattractors are other special points in the bifurcation diagram. These are states of the highest asymptotic stability. Each phase has its own superattractor. In the bifurcation diagram (Figure 3(a)), the superattractors are shown by small white circles. Due to their superstability, superattractors are states in which the vast majority of the vacuum cells prefer to dwell, the ground states.

Phase $0(B<-0.5)$ is a special phase. Perhaps this phase is more relevant to cosmology. In Phase 0 , the amplification parameter is $A<1$, and it describes feedback-loop attenuation. Selfgravitation and self-diffusion microflows penetrate each other with weak interactions and are incapable of vortex (vacuum cell) formation.

The dust component, associated with matter, tends to disperse under prevailing self-diffusion. It disperses with acceleration when dust evolves toward the superattractor, $B=-1$. Its evolution supports the phenomenon of galaxies receding from each other, typically attributed to the positive cosmological constant $\Lambda$ or dark energy.

The dust component, associated with antimatter, under prevailing self-gravitation, evolves toward bifurcation at $B=-0.5$. During its evolution it slows down the convergence rate, which becomes infinitively slow at the point of bifurcation. Below, we will see that time dilation is universal for vacuum cellular networks moving toward bifurcations. It is as universal as its relativistic counterpart. However, instead of gravitational collapse or formation of black holes, at bifurcation $B=-0.5$, the medium forms vacuum cells.

This happens when the amplification parameter $A$ reaches a critical value $A=1$. The iteration feedback loops are amplified, and the medium becomes active. In active media, vortex formation is ubiquitous. It is observed in the form of various turbulent flows, such as atmospheric tornados, solar protuberances, or galaxy formations. Perhaps the most relevant to the proposed model are the Bénard-cells [1], formed in thermodynamically nonequilibrium gases and liquids. Vacuum cells (vortices) emerge when interactions between the competing antiparallel dust flows lose tangential stability and tangential fluctuations are amplified until they form circular flows. The vortices are dynamic (not thermodynamic) equilibrium states that possess asymptotic stability and evolve toward their attractors. The convergence rate toward attractors (which is a measure of their asymptotic stability) is different for each $B$, and maximal at the superattractor $(B=0)$.

In Phase 1, an attractor represents a single closed loop in phase space (winding number $\mathcal{W}_{1}=$ 1, Figure 4) that implies $U(1)$ symmetry. There are two branches in the bifurcation diagram, one for cells (blue) and the other for anti-cells (red). They cross each other at the superattractor $B=0$, where generic charge vanishes $\chi_{\infty}=0$ (small white circle in the bifurcation diagram, Figure 3(a)). The superattractor collects the vast majority of cells, and on average, they are charge-neutral. We associate Phase 1 cellular networks with the electromagnetic field.

The next bifurcation at $B=0.5$ is a phase transition to Phase 2. Here, the cell dynamics become more complex. Iterated maps (7) lose stability and oscillate between two different charge states 
depicted in the bifurcation diagram by two branches for cells and two branches for anti-cells. To avoid ambiguity, iteration function (5) is replaced with two double-iterated iteration functions having the same explicit form

$$
\mathcal{L} 2(\chi, B)=\mathcal{L}(\mathcal{L}(\chi, B))=B-(1+B)\left(B-(1+B) \chi^{2}\right)^{2},
$$

but initiated at different starting points. The first iteration starts at any point $\chi_{0,1}$ from the domain $|\chi|<1$. The second iteration does not have this freedom and starts at $\chi_{0,2}= \pm \mathcal{L}\left(\chi_{01}, B\right)$. Iteration functions (8) are second-degree polynomials in $\chi^{2}$ that live in a complex two-dimensional phase space $\mathbb{C}^{2}$. The attractors acquire second loops (winding number $\mathcal{W}_{2}=2$, Figure 4 ). As in Phase 1 , two branches (one cellular and one anti-cellular) cross each other at the superattractor $(B \approx 0.618)$ at a charge-neutral state $\left(\chi_{\infty}=0\right.$, small white circle in Figure 3(a)), while the other two branches have complimentary charges (small purple circles in Figure 3 top).

Two-loop attractors possess some spinorial features in the sense that they live in $\mathbb{C}^{2}$ phase space and require two full rotations $\left(720^{\circ}\right)$ to return to their initial states. We call a vacuum cell spinorial if its attractor has two or more loops $(\mathcal{W} \geq 2)$. Spinorial cells and anti-cells are particle building blocks. We associate Phase 2 cellular networks with the weak nuclear field, which possess $S U(2)$ symmetry. The superattractor Phase 2 charged states are associated with electron charge (cell) and positron charge (anti-cell).

Below we will discuss spinorial cell synchronization patterns with the help of circular time diagrams (CTDs). CTDs represent the charge state a given vacuum cell (or anti-cell) belongs to. For Phase 2 spinorial cells, CTDs are shown in Figure 5.

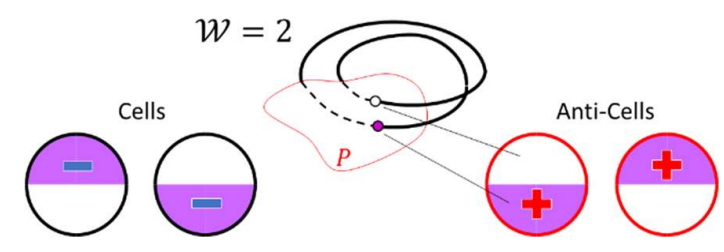

Figure 5. Phase 2 circular time diagrams (CTDs). Spinorial cells are marked by black rims (left) and anticells marked by red rims (right). The neutral states are represented by white sectors and the charged states by purple sectors. The diagrams rotate in time with cellular period $\mathcal{T}$. The current charge states are represented by top sectors.

The next period-doubling bifurcation brings vacuum cells to Phase 3. The iterated map with double-iterated iteration functions (8) lost stability and is replaced by four new iteration functions, this time four-times iterated the original one

$$
\begin{gathered}
\mathcal{L} 3(\chi, B)=\mathcal{L}(\mathcal{L}(\mathcal{L}(\mathcal{L}(\chi, B)))) \\
=B-(1+B)\left(B-(1+B)\left(B-(1+B)\left(B-(1+B) \chi^{2}\right)^{2}\right)^{2}\right)^{2} .
\end{gathered}
$$

The first iteration starts at any point $\chi_{0,1}$ from the domain $|\chi|<1$. The starting points for the other iterated functions are at $\chi_{0, k}= \pm \mathcal{L}\left(\chi_{0, k-1}, B\right), k=2,3,4$.

$\mathcal{L} 3$ s are eighth-degree polynomials in $\chi^{2}$ and live in eight-dimensional complex space $\mathbb{C}^{8}$. The attractor winding number is $\mathcal{W}_{3}=4$. Two out of eight branches on the bifurcation diagram (one cellular and one anti-cellular) cross each other at the superattractor $(B \approx 0.7493)$ at a charge-neutral state (white circle). The other six (three cellular and three anti-cellular) are charged and depicted on the bifurcation diagram by the red, green, and blue circles. We associate Phase 3 cellular networks 
with the strong nuclear field, which possess $S U(3)$ symmetry and is mediated by eight gluons carrying three color charges and three conjugated color charges.

CTDs for Phase 3 spinorial cells are shown in Figure 6.

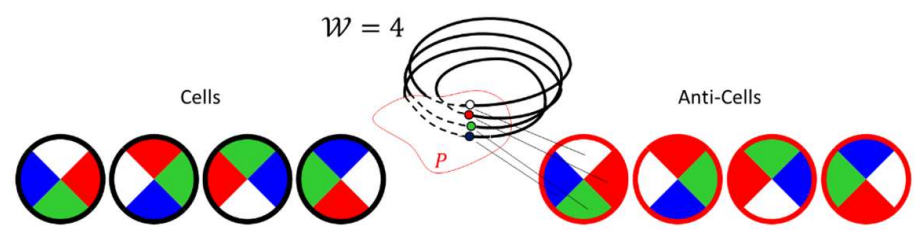

Figure 6. Phase 3 circular time diagrams for spinorial cells. Cells are marked by black rims (left) and anticells are marked by red rims (right). Three color sectors represent charged states. The white sector state is neutral. Diagrams rotate in time counterclockwise with the period equal to the total cellular period $\mathcal{T}$. The current charge state is represented by the top sectors.

The direction of cellular evolution (order of charged states) is fixed. It is the same for cells and anti-cells, and cannot be reversed (due to time irreversibility):

$$
\text { ... white } \rightarrow \text { red } \rightarrow \text { green } \rightarrow \text { blue } \rightarrow \text { white } \ldots
$$

Correspondingly, diagrams rotate in time in one direction only (counterclockwise). This is crucial for the following discussion of quark mixing amplitudes and CKM-matrix features.

The proposed model does not preclude the existence of cellular networks beyond Phase 3 . However, at this time we are not ware of any natural objects that can be associated with them. Thus, they are beyond the scope of this paper.

\section{Asymptotic Stability. $\lambda$-wells. Universal Time Dilation}

Asymptotic stability is a major characteristic of cellular dynamics. The Lyapunov exponent $\lambda$ is a measure of asymptotic stability. We calculate the Lyapunov exponents using evolutionary trajectories $\chi_{n}(B)$ that are converging toward their attractors $\chi_{\infty}(B)$ as

$$
\lambda(B)=\lim _{n \rightarrow \infty}\left(\ln \left|\frac{\chi_{n+1}(B)-\chi_{n}(B)}{\chi_{n}(B)-\chi_{n-1}(B)}\right|\right) .
$$

$\lambda(B)$ is shaped as a set of wells filling $B$-space between bifurcations (Figure 3(b)). $\lambda$-well widths (phase space sizes) progressively decrease from phase to phase with the scaling factor quickly converging toward the universal constant $\delta_{F}=4.669 \ldots$ (the Feigenbaum delta).

At bifurcations, $\lambda=0$ and manifests conditional cellular stability. It is a state at the boundary between two different phases. To persist at a bifurcation, a cell needs to be isolated from the rest of the world, i.e. be conserved. Otherwise, any small perturbation will spontaneously break the state symmetry and move the cell to one or the other phase, to the states of higher stability (where $\lambda$ is negative). Which phase a cell selects to remain in, depends on the perturbation (a spontaneously broken symmetry). All other states are asymptotically stable $(\lambda<0)$. Superattractors possess the highest asymptotic stability $(\lambda \rightarrow-\infty)$.

Phase 1, Phase 2, and Phase $3 \lambda$-wells have similar (but not identical) shapes. Their walls are almost logarithmic. Phase 2 and Phase $3 \lambda$-wells can be approximately described as elevated Phase $1 \lambda$-wells. All three wells can be described as

$$
\lambda_{j}(\Delta B) \approx \ln |2 \Delta B|+(j-1) \ln \delta_{F},
$$


where $j$ is the phase number, $\Delta B$ is the departure from superattractor $B_{j S}$, and

$$
\Delta B=B-B_{j S}
$$

Parameter $\lambda$ is connected to the convergence time constant (a measure of cellular relaxation speed), which in Phase $j$ is

$$
\tau_{j}(B)=-\frac{\mathcal{T}}{\lambda_{j}(B)}
$$

where $\mathcal{T}$ is the cellular (vortex) rotation period. Time constant $\tau_{j}(B)$ in $\mathcal{T}$-units is shown in Figure 7.

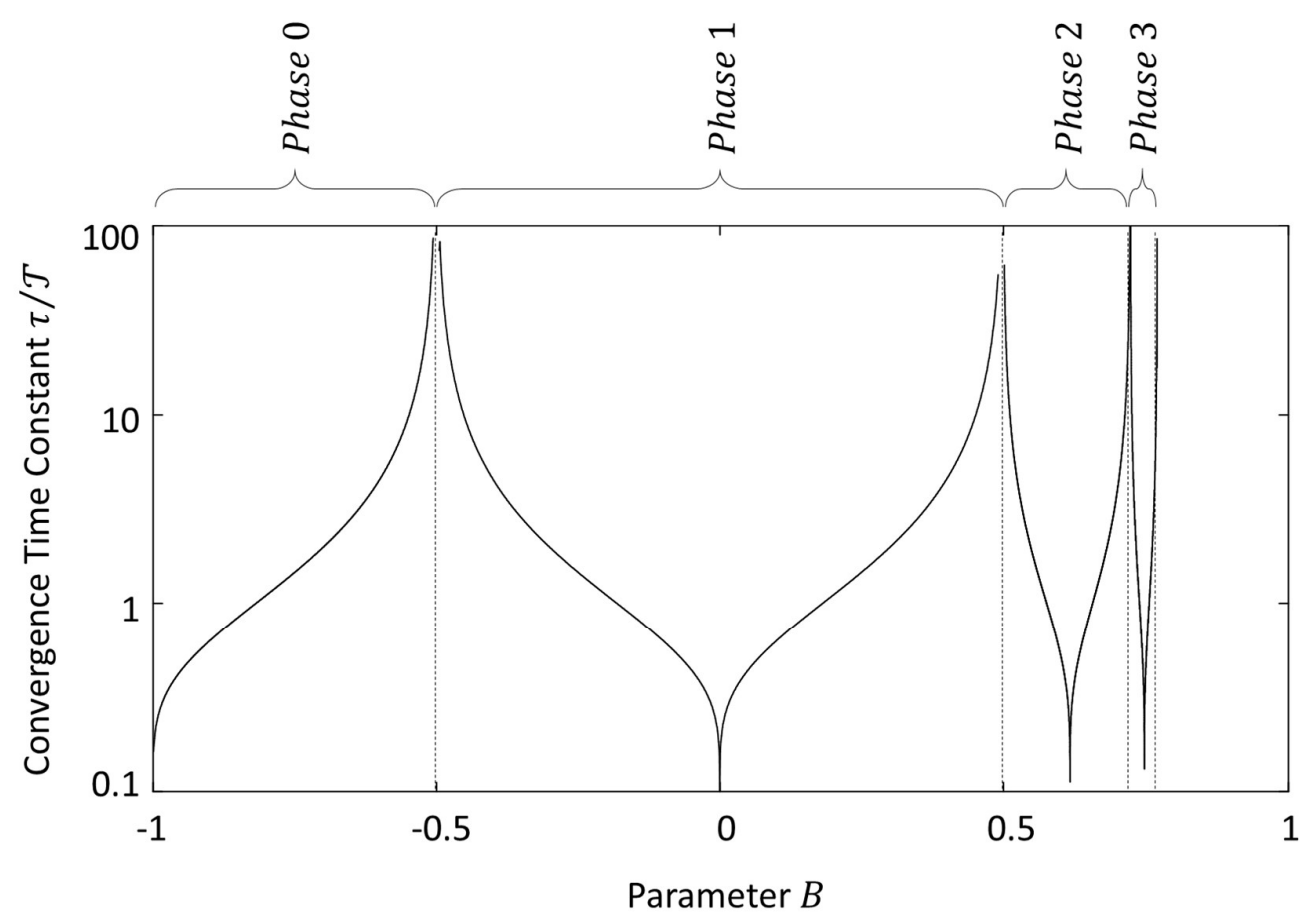

Figure 7. Reduced convergence time constant $\tau_{j}(B) / \mathcal{T}$

For intracellular dynamics, period $\mathcal{T}$ is an internal unit of time. In synchronized cellular networks, $\mathcal{T}$ is promoted to the universal time standard for the entire network. However, $\mathcal{T}$ cannot be measured by just any imaginable clock because every device consists of several vacuum cells that cannot operate coherently until their intracellular relaxation is complete. The intracellular relaxation time limits the rate of ideal clocks. Therefore, clocks operate faster at superattractors and slow down near bifurcations. This time dilation applies to every device, field, and even elementary particles because (according to the proposed model) all of them, including leptons and quarks (see below), consists of at least two vacuum cells. This universality is in accordance with the universality of relativistic time dilation.

\section{Cellular Thermodynamics.}

$\lambda$-wells resemble potential wells of a bound system. We extend this similarity and define cellular energy as 


$$
\varepsilon=\frac{\lambda}{\mathcal{T}}
$$

In a system of units where $\mathcal{T}=1$, energy $\mathcal{E}(B)=\lambda(B)$ (Figure $3(\mathrm{~b})$ ).

From (12) and (15), the energy in Phase $j(j=1,2,3)$ is described as

$$
\varepsilon_{j}(\Delta B)=\frac{\ln |2 \Delta B|+(j-1) \ln \delta_{F}}{\mathcal{T}}
$$

or

$$
\varepsilon_{j}(\Delta B)=\frac{\ln |2 \Delta B|}{\mathcal{T}}+\mu_{j}=\varepsilon_{1}(\Delta B)+\mu_{j}
$$

where we define cellular chemical potential in Phase $j$ as

$$
\mu_{j}=\frac{(j-1) \ln \delta_{F}}{\mathcal{T}}
$$

A perturbation can kick a cell from one evolutionary trajectory to another, including trajectories that belong to a different attractor basin. The higher the ambience temperature, the frequently the trajectory switches occur. We define the system temperature $\theta$ as reciprocal to the average time $t_{\theta}$ a cell spends at a single evolutionary trajectory

$$
\theta=\frac{1}{t_{\theta}}
$$

Using definitions (15) - (19), we can estimate cellular distribution across energies.

Evolution at a new trajectory can start at any state $\left(\chi_{0}, B\right)$ and proceed toward its attractor with time constant provided by equation (13). Using (17), the time constant is

$$
\tau_{j}(\Delta B)=-\left(\varepsilon_{1}(\Delta B)+\mu_{j}\right)^{-1} \text {. }
$$

We define distance $\Delta \chi_{n}$ from a fixed point $\chi_{\infty}$ after the $n$-th iteration as

$$
\Delta \chi_{n}=\left|\chi_{n}-\chi_{\infty}\right| \text {. }
$$

During a cell's evolution along a single trajectory, it exponentially decreases with time (iteration steps) as shown in Figure 8 for three different $B$-values and different starting points $\chi_{0}$. 


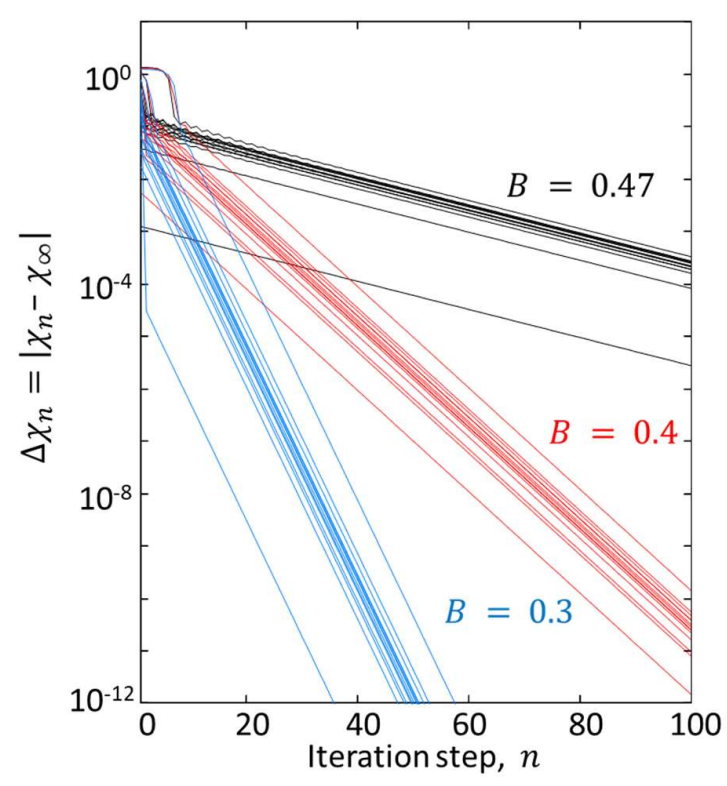

Figure 8. $\Delta \chi$-evolution for different parameters $B$ (different colors) and different starting points $\chi_{0}$.

All trajectories belonging to the same parameter $B$ but starting at different initial points are covered by an envelope $\Delta \mathrm{X}(B, t)$ (Figure 9), which shrinks exponentially with the same time constant as the trajectories it embraces

$$
\Delta \mathrm{X}(B, t) \propto \exp \left(t\left(\varepsilon_{1}(|\Delta B|)+\mu_{j}\right)\right) .
$$

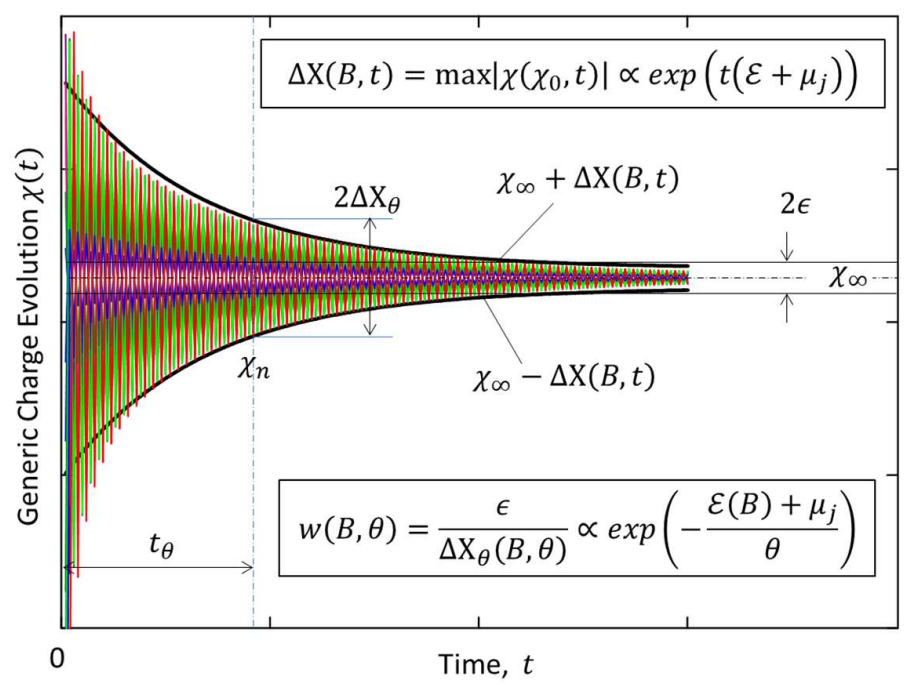

Figure 9. Evolution of envelope $\Delta \mathrm{X}(B, t)$ that enfolds $\chi(t)$ trajectories belonging to the same parameter $B$.

At temperature $\theta$, the average evolutionary time is $t=t_{\theta}$, and trajectories converging toward attractor $B$ are inside interval $\Delta \mathrm{X}_{\theta}(B)$ 


$$
\Delta \mathrm{X}_{\theta}(B) \propto \exp \left(\frac{\mathcal{E}_{1}(\Delta B)+\mu_{j}}{\theta}\right)
$$

Assuming uniform trajectory distribution inside the envelope $\Delta \mathrm{X}_{\theta}$, the probability $w$ to find a cell inside a small fixed interval $2 \epsilon$ is

$$
w(B, \theta) \propto \frac{\epsilon}{\Delta \mathrm{X}_{\theta}(B)} .
$$

Assuming that cells belong to a single phase, after substitution of (23) into (24), we obtain the cellular partition function. In Phase $j$ this probability is

$$
w_{j}(\Delta B, \theta)=\frac{1}{N_{j}(\theta)} \exp \left(-\frac{\mathcal{E}_{1}(\Delta B)+\mu_{j}}{\theta}\right),
$$

where the normalization factor is

$$
N_{j}(\theta)=\int_{B_{j L}}^{B_{j R}} \exp \left(-\frac{\mathcal{E}_{1}(\Delta B)+\mu_{j}}{\theta}\right) d \Delta B .
$$

Integral (26) is taken across Phase $j$, from the left-sided bifurcation $B_{j L}$ through the right-sided bifurcation $B_{j R}$. Partition functions (25) have the form of a Boltzmann distribution (although cells are bound states thus energies are negative). To escape infinite values, we limit the relaxation time to values $\tau \gtrsim \mathcal{T} / 3$, that corresponds to 95\%-convergence during one cellular period. Respectively, the Lyapunov exponents are $\lambda \gtrsim-3$, and energies are $\mathcal{E} \gtrsim-3 / \mathcal{T}$.

We define the critical temperature of cell dissociation $\theta_{c}$ as the temperature when cellular evolution time at a single trajectory becomes as short as the cellular period

$$
\theta_{c}=\mathcal{T}^{-1}
$$

A few examples of cellular distributions across energies for different temperatures in different phases are shown in Figure 10. Departure from the critical temperature quickly narrows the partition function, which starts to resemble Dirac's delta-function.

Even at moderate temperatures, the vast majority of cells are located at the superattractors. This is the reason why generic charges are quantized and their superattractor values become physical constants.

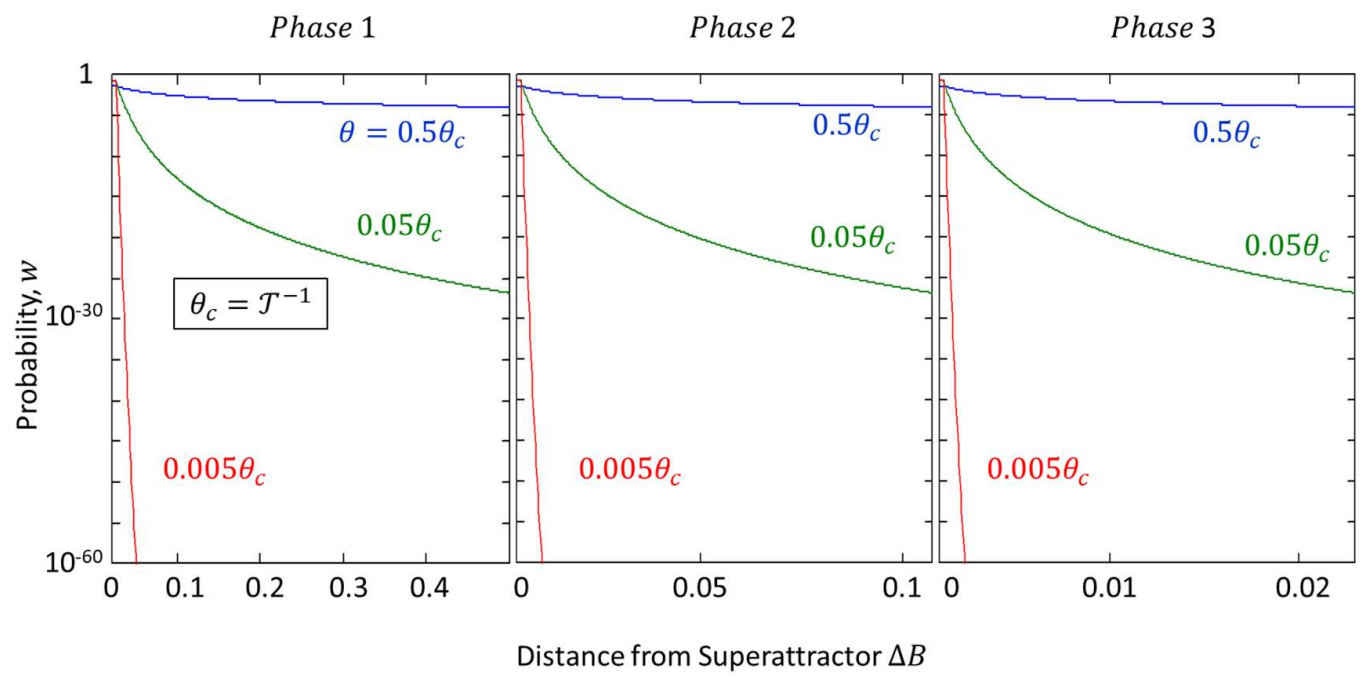


Figure 10. Cellular distributions at high temperatures

Most time cells spend at superattractors (ground sates). A cell briefly departed from a superattractor state (Figure 11(b)) can be compared to a virtual particle briefly departed from a mass shell (Figure 11(a)).
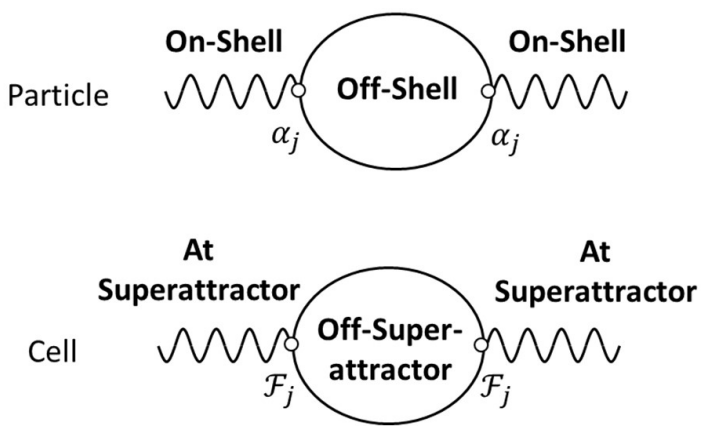

Figure 11. Feynman-like self-interaction diagrams for: (a) particle; (b) cell.

To extend this parallel, we introduce restoring forces acting on excited cells and pushing them toward the superattractors. The strength of the restoring forces plays the role of the coupling constants in the processes encoded in Figure 11(b). The restoring forces (in Phase $j$ ) are defined as

$$
\mathcal{F}_{j}=-\frac{\partial \varepsilon_{j}}{\partial \Delta B}
$$

By taking the derivative of (17), (28) can be written as

$$
\mathcal{F}_{j}=-\frac{1}{\mathcal{T} \Delta B} .
$$

The restoring forces are strongest at the superattractors and monotonically soften when a cell departs from a superattractor, thus resembling asymptotic stability of color forces.

From (17) and (29), the dependence of restoring forces on energy or the Lyapunov exponent, in Phase $j$ is

$$
\mathcal{F}_{j} \approx \mp \frac{2 \delta_{F}^{j-1} \exp \left(-\varepsilon_{j} \mathcal{T}\right)}{\mathcal{T}}=\mp \frac{2 \delta_{F}^{j-1} \exp \left(-\lambda_{j}\right)}{\mathcal{T}}
$$

where the force is directed toward the corresponding superattractor (by the proper selection of its sign "+" or "-"). According to (30), at a fixed energy, the restoring forces in different phases relate to each other as

$$
\mathcal{F}_{1}: \mathcal{F}_{2}: \mathcal{F}_{3} \approx 1: \delta_{F}: \delta_{F}{ }^{2} \approx 1: 4.67: 21.8,
$$

where $\delta_{F}$ is the Feigenbaum delta.

For comparison, the relation among the SM field coupling constants at energy $\sim 80 \mathrm{GeV}-90 \mathrm{GeV}$ is

$$
\alpha: \alpha_{w}: \alpha_{s} \approx 1: 4.32: 15.1 \text {. }
$$

The numbers on the right side of (32) are obtained using CODATA source [8]: 
$\alpha\left(m_{Z}\right) \approx 1 / 128 \approx 0.00781 ; \sin ^{2} \theta_{W}\left(m_{W} / m_{Z}\right)=\frac{\alpha}{\alpha_{w}} \approx 0.231 ; \alpha_{S}\left(m_{Z}\right) \approx 0.1182 ; m_{Z} \approx 91.2 \mathrm{GeV} / \mathrm{c}^{2}$ $m_{W} \approx 80.4 \mathrm{GeV} / \mathrm{c}^{2}$

When temperature increases above the critical value $\theta_{c}$, vacuum cells dissociate. On a back process of condensation, the relative probability $W_{k} / W_{l}$ of finding a cell in Phase $k$ or Phase $l$ is determined by the

$$
\frac{W_{k}}{W_{l}}=\exp \left(\frac{\mu_{l}-\mu_{k}}{\theta_{c}}\right)=\delta_{F}^{l-k},
$$

In a two-component system, the total probability obeys unitary condition

$$
W_{l}+W_{k}=1
$$

This allows us to represent the probabilities $W_{k}$ and $W_{l}$ as trigonometric functions of a single parameter, which we call mixing angle $\gamma_{k l}$

$$
\left\{\begin{array}{l}
W_{k}=\sin ^{2} \gamma_{k l} \\
W_{l}=\cos ^{2} \gamma_{k l}
\end{array}\right.
$$

Correspondingly,

$$
\tan ^{2} \gamma_{k l}=\exp \left(\frac{\mu_{l}-\mu_{k}}{\theta_{c}}\right) .
$$

Specifically, for the Phase 1/Phase 2 mixture, the mixing angle

$$
\gamma_{21}=\operatorname{atan}\left(\sqrt{W_{2} / W_{1}}\right)=\operatorname{atan}\left(\delta_{F}^{-1 / 2}\right) \approx 24.8^{\circ},
$$

which is close to the Weinberg mixing angle $\theta_{W} \approx 28.7^{\circ}$ [9] that reflects the relative inputs of electromagnetic and weak forces in the combined electro-weak interactions.

\section{Intercellular Dynamics. Synchronization}

Synchronization $[10,11]$ is a ubiquitous dissipative phenomenon observed in all types of active systems: natural and artificial, organic and inorganic, in mechanical, chemical, and electronic oscillators that are similar and dissimilar to one another, with different structures, organizations, and waveforms, including chaotic waveforms. Vacuum cells are open systems that couple to their neighbors via dust particle exchanges. As coupled self-sustained oscillators (rotators), they tend to synchronize their dynamics. Before synchronization, their rotation periods are different. Synchronization bring them to the same period $\mathcal{T}$, which becomes a time-scale standard for the entire synchronized network.

Synchronization is a phase transition, which not only creates a unified time scale, but provides temporal coherency to the medium (crucial for quantum theory). As a phase transition, synchronization creates a new order parameter, the phase differences between vacuum cells $\psi^{\alpha \beta}=\varphi^{\alpha}-\varphi^{\beta}$, which plays the role similar to a vector potential in a gauge field. Synchronization is a nonlinear process, which includes positive feedback loops and facilitates the transformation of cellular partition function (25) into quantum distributions.

To illustrate synchronization between two vacuum cells (designated as $\alpha$-cell and $\beta$-cell), we use the Adler phase-difference equation [12], which we converted to a discrete-time iterated map: 


$$
\psi_{n+1}=\psi_{n}-\delta \omega \mathcal{T}+\mathrm{K} \sin \psi_{n}
$$

where $\delta \omega$ is the difference between cellular natural frequencies $\delta \omega=\omega_{\alpha}-\omega_{\beta} ; \omega_{\alpha}=\mathcal{T}_{\alpha}^{-1}$ and $\omega_{\beta}=$ $\mathcal{J}_{\beta}^{-1}$ are natural frequencies that characterize cells before synchronization; $\psi_{n}=\varphi_{n}^{\alpha}-\varphi_{n}^{\beta}$ is the intercellular phase difference after the $n$-th iteration step and $K$ is the coupling strength between the cells. Because both cells converge to the same period, the iteration time interval can be associated with any of the coupled cells, for example, $\alpha$-cell. The corresponding cellular phase is set to $\varphi_{n}^{\alpha} \equiv 0$.

Phase-difference dynamics are illustrated in Figure 12. The phase entrainment indicates that $\psi_{n}$ converges toward a fixed point $\psi_{\infty}=\psi$. This occurs when $|\delta \omega \mathcal{T}|<K$. Within the phase-entrainment interval, the correspondence between the old parameter $\delta \omega$ and the new order parameter $\psi$ is oneto-one.

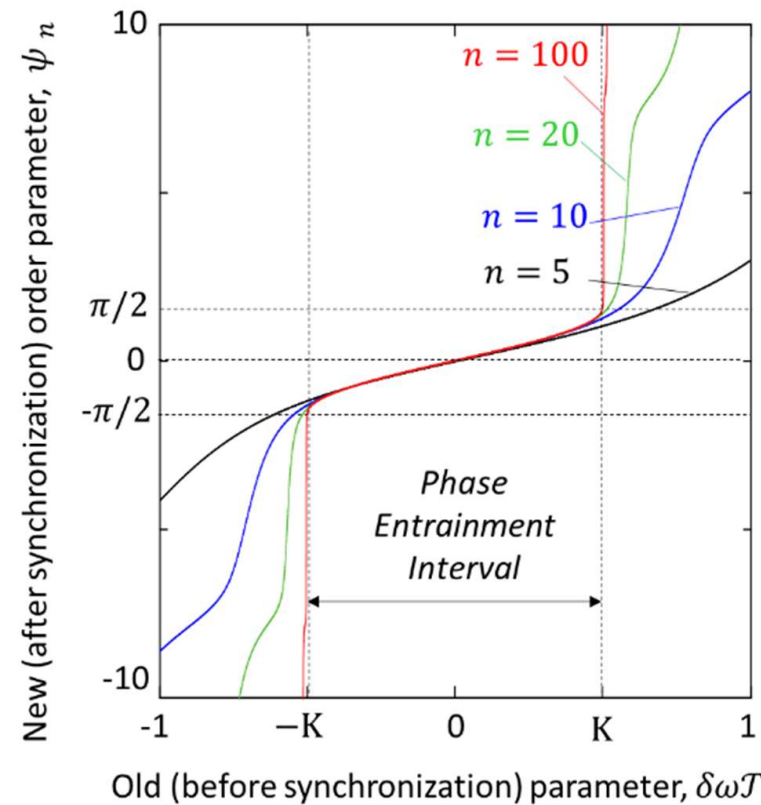

Figure 12. Phase differences $\psi_{n}$ after $n$ iteration steps

Synchronization is a dissipative process that possess asymptotic stability. We calculate the Lyapunov exponent $\lambda$ in a similar fashion to (11), where parameter $B$ is replaced by parameter $\psi$ :

$$
\lambda(\psi)=\lim _{n \rightarrow \infty}\left(\ln \left|\frac{\psi_{n+1}(\psi)-\psi_{n}(\psi)}{\psi_{n}(\psi)-\psi_{n-1}(\psi)}\right|\right) .
$$

We simulate functions $\lambda_{j}(\psi)$ and $\mathcal{E}_{j}(\psi)$ assuming that 1) coupling constants in different phases $K_{j}$ are proportional to the intracellular restoring forces $\mathcal{F}_{j}$ in the corresponding phases (see (31)), and 2) intracellular coupling is strong in Phase $3\left(K_{3} \lesssim 1\right)$. The simulation results for $K_{1}=$ $\delta_{F}{ }^{-2}, K_{2}=\delta_{F}^{-1}$, and $K_{3}=0.999$ are shown in Figure 13. 

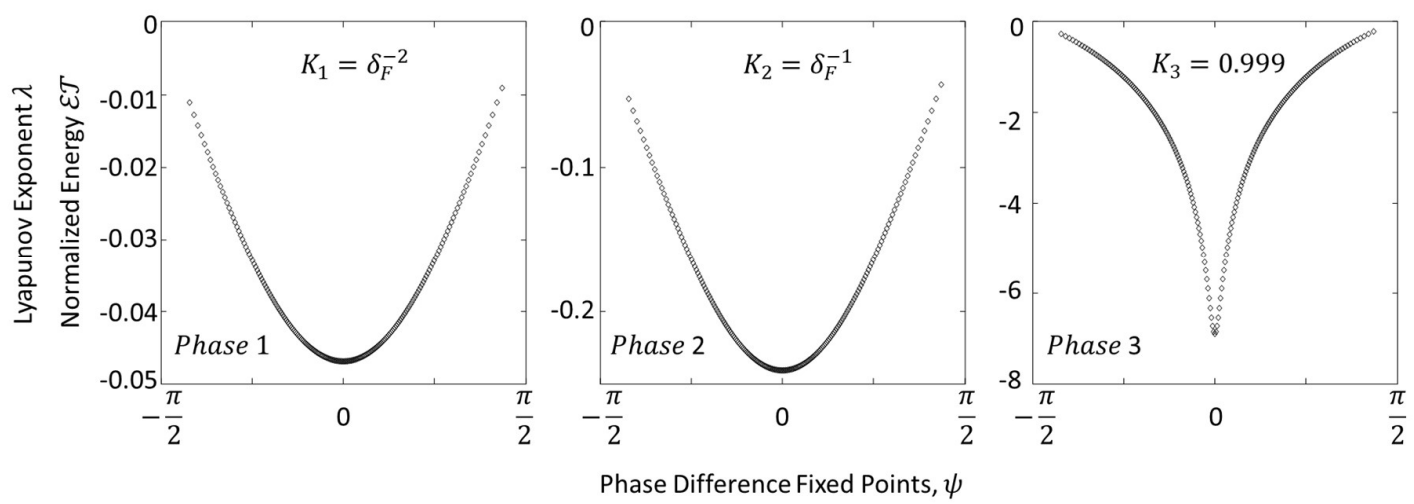

Figure 13. $\lambda_{j}(\psi)$-wells and normalized $\mathcal{T} \mathcal{E}_{j}(\psi)$ potential wells for synchronized couples belonging to different phases.

As in the intracellular case, the graphs are shaped as potential wells with their minima at the superattractors. Here couples operate in unison $(\psi=0)$. The well shapes are qualitatively different between the weak intercellular coupling (Phase 1 and Phase 2) and the strong intercellular coupling (Phase 3). In Phase 3, the well shape is similar to that of the intracellular dynamics previously shown in Figure 3(b).

We compare the Phase 3 intercellular and intracellular barriers in Figure 14.

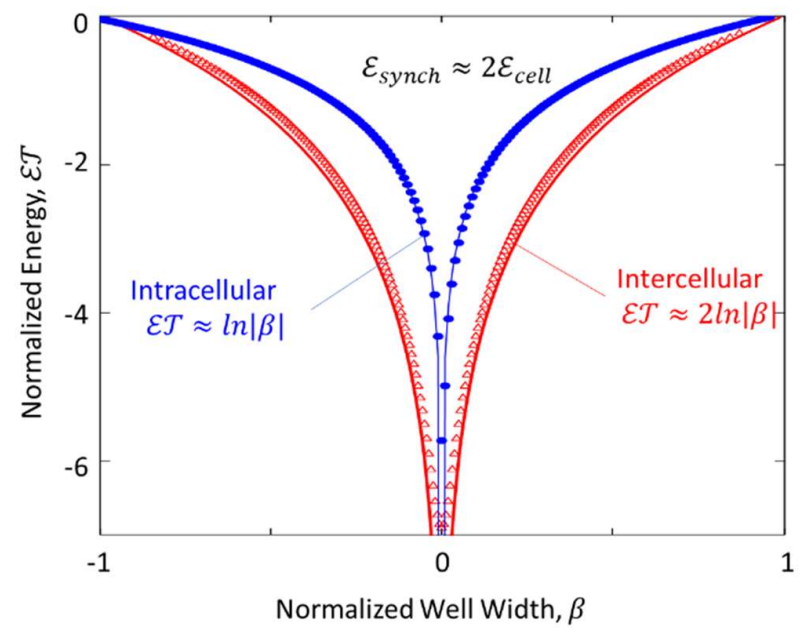

Figure 14. Comparison of Phase 3 intracellular (blue) and intercellular (red) potential barriers. Simulated points are shown by symbols. Logarithmic curves are shown by solid lines.

The intercellular (synchronization) potential barrier $\varepsilon_{\text {synch }}(\beta)$ is drawn in red and the intracellular potential barrier $\mathcal{E}_{\text {cell }}(\beta)$ is drawn in blue. Parameter $\beta$ is the normalized departure from a superattractor. It is defined as

$$
\beta=\left\{\begin{array}{ll}
2 \frac{B-B_{3 S}}{B_{3 R}-B_{3 L}} & \text { (intracellular) } \\
2 \frac{\psi}{\pi} & \text { (intercellular) }
\end{array},\right.
$$


where $B_{3 S}, B_{3 L}$, and $B_{3 R}$ are positions of the Phase 3 superattractor, the left bifurcation, and the right bifurcation, respectively. At superattractors, $\beta=0$, and at bifurcations, $\beta= \pm 1$. The well walls are approximated by logarithmic curves (solid lines in Figure 14). According to these data, when coupling between cells is strong, the coupling energy is comparable to the total internal energy of both cells

$$
\varepsilon_{\text {synch }} \approx 2 \varepsilon_{\text {cell }} \text {. }
$$

We will use (41) for estimating of quark transmutation probabilities.

In Phase 1 and Phase 2, the well bottoms are parabolic (Figure 13 left and center), which implies linearity of the restoring forces and synchronized network elasticity. We define intercellular restoring forces $\mathfrak{f}_{j}$ similarly to their intracellular counterparts (28)

$$
f_{j}=-\frac{\partial \varepsilon_{j}}{\partial \psi}
$$

and calculated functions $f_{j}(\psi)$ (Figure 15).
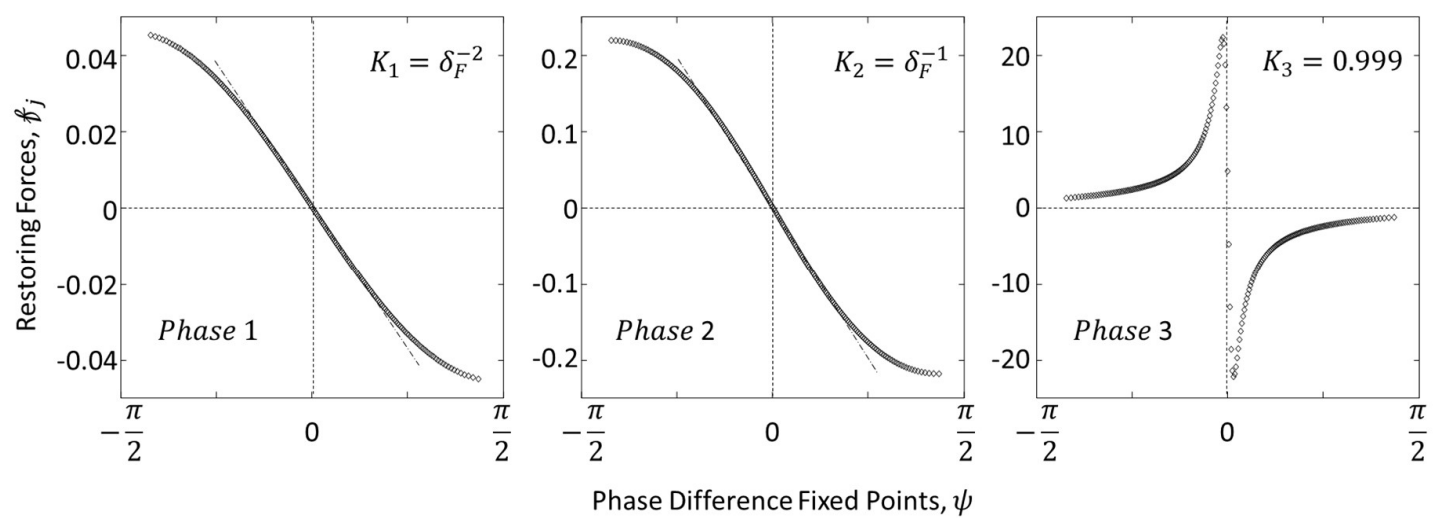

Figure 15. Restoring forces $f_{j}(\psi)$ in potential wells attributed to different phases imply cellular network elasticity in Phase 1 and Phase 2 and asymptotic freedom in Phase 3.

In Phase 1 and Phase 2, near superattractors $(\psi \sim 0)$, the restoring forces linearly increase with departure from superattractors and saturate near the bifurcations $(\psi \sim \pm \pi / 2)$. In these two intervals, at fixed phase differences $\psi=$ const, the restoring forces are related as follows

$$
f_{1}: f_{2} \approx K_{1}: K_{1} \approx 1: \delta_{F}
$$

In Phase 3 (strong coupling), the restoring force behaves quite differently. The interval of linearity (if any) is very narrow (it disappears when the coupling parameter is exactly $K_{3}=1$ ). When departing from the superattractor, $f_{3}$ almost immediately reaches its large and sharp maximum then quickly decays, mimicking the phenomenon of color-force asymptotic freedom.

The intercellular dynamics discussed above are extendable to synchronized cellular networks, where each cell is surrounded by several others. In this case, $\mathcal{E}$ and $f$ correspondingly become tensors.

Synchronized cellular networks also possess quantum features. During synchronization, each coupled cell forces its neighbors to operate at its pace. After iterative "negotiations", the cells come to a trade-off period. The restoring forces push cells to operate not only with the same period but also at the same phase (in unison). If two or more cells from a cell neighborhood operate at the same 
phases, they multiply their efforts to force other cells to operate at the same phase. This nonlinearity implies a positive feedback: the more cells in a network operate at a given phase, the higher the probability to find another cell at the same phase. As a result, the random distribution across phases spontaneously breaks and a discrete set of superattractors emerges at positions of seed states, as schematically is shown in Figure 16.

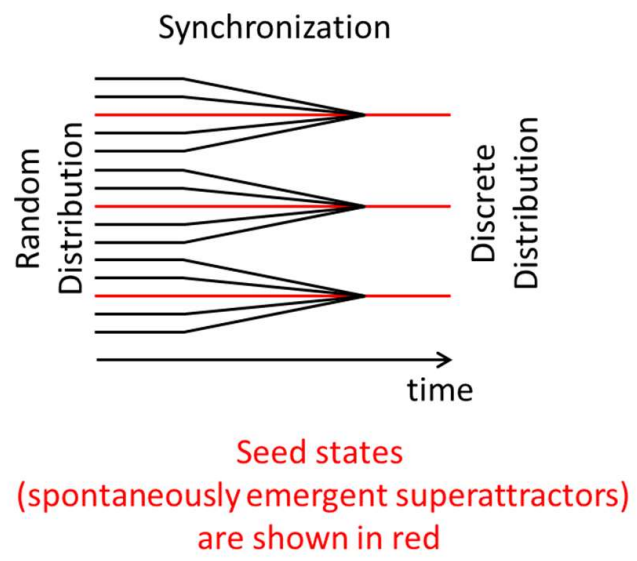

Figure 16. Synchronization positive feedback transforms random cellular distribution into quantum distribution.

Synchronization alters the Boltzmann-like partition function (25). Synchronization can occur according to several scenarios. Below, we will consider two of them: a case of phase entrainment (qualitatively described above) and a case of oscillation quenching [10,13, 14]. Which scenario occurs in a network depends on details that are beyond the scope of this paper. Below, we substitute phase differences with energies.

Phase-entrainment scenario. Let us consider a group of cells, some of which have been synchronized at states with energy $\mathcal{E}$ ( $\mathcal{E}$-state). The reminders have similar but nonidentical energies. The synchronized cells force the other cells to move to the $\mathcal{E}$-state. Let us assign the initial probability of finding a cell at the $\mathcal{E}$-state to be $w$, and the resulting probability to be $w_{+}$. In a simplest case, the probability to get a new cell to the same state is proportional to the number of cells at this state $\kappa_{+} w_{+}$, where $\kappa_{+}$is the proportionality coefficient. The total number of new cells is $\kappa_{+} w_{+} w$, and the total number of all cells at the $\mathcal{E}$-state after the new cells have been added is

$$
w_{+}=w\left(1+\kappa_{+} w_{+}\right) .
$$

The " +" signs in (44) indicate that synchronization occurs according to the phase-entrainment scenario.

After solving equation (44) for $w_{+}$,

$$
w_{+}=\frac{1}{\frac{1}{w}-\kappa_{+}},
$$

and substituting (25) in (45), the altered partition function takes on the form of the Bose-Einstein distribution 


$$
w_{+, j}(\varepsilon, \theta)=\frac{\kappa_{+}^{-1}}{\left(\kappa_{+} C_{0}\right)^{-1} \exp \left(\frac{\mathcal{E}_{1}+\mu_{j}}{\theta}\right)-1}
$$

Oscillation (rotation) quenching scenario. According to this scenario, cellular interactions result in the mutual annihilation of circular flows, resulting in the disappearance of coupled cells from the network. This phenomenon is also known as amplitude death or oscillation cessation [10, 13, 14]. Here, the competition occurs between the network cells and their environment, which tends to destroy the network. In this case, the positive feedback of synchronization means that the higher the number of cells which are mutually annihilated, the weaker the network, and the higher the chance that a different cell will also fails to withstand this environment.

To find the cellular distribution under the oscillation quenching scenario, we use equations similar to (44) but with all the " + " signs replaced with " - " signs

$$
w_{-}=w\left(1-\kappa_{-} w_{-}\right) \text {. }
$$

After solving (47) for $w_{-}$

$$
w_{-}=\frac{1}{\frac{1}{w}+\kappa_{-}},
$$

and substituting (25) in (48), the altered partitions function takes on the form of the Fermi-Dirac distribution

$$
w_{-, j}(\mathcal{E}, \theta)=\frac{\kappa_{-}^{-1}}{\left(\kappa_{-} C_{0}\right)^{-1} \exp \left(\frac{\mathcal{E}_{1}+\mu_{j}}{\theta}\right)+1} .
$$

According to the above scenarios, positive feedback stemming from synchronization converts the original distribution of independent cells into quantum distributions. Unlike in the case of quantum statistics, quantization from synchronization does not require cellular identity.

\section{Synchronized Spinorial Cells as Particle Building Blocks}

Spinorial cells (Phase 2 and Phase 3) occupy different charge states during each cellular period (as illustrated by the attractor-loop diagram in Figure 17). The white loops represent quasi-neutral states, the purple loop represents electrically charged states, and red, green, and blue loops represent color-charged states. The charge states are ordered in time. Color-charge states always follow the same sequence: ... $\rightarrow$ neutral $\rightarrow$ red $\rightarrow$ green $\rightarrow$ blue $\rightarrow$ neutral $\rightarrow \ldots$ in one direction.

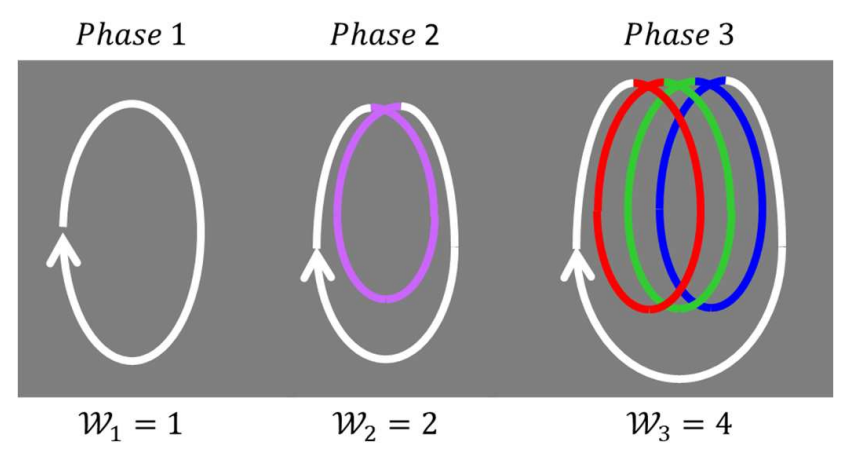

Figure 17. Attractor loop diagrams. Charge-color code is the same as in Figures 3, 5, and 6 
Below, we will neglect small cellular departures from superattractors, and describe connected spinorial couples as in-phase synchronized and out-of-phase synchronized, only keeping in mind their loops. Examples are shown in Figure 18.

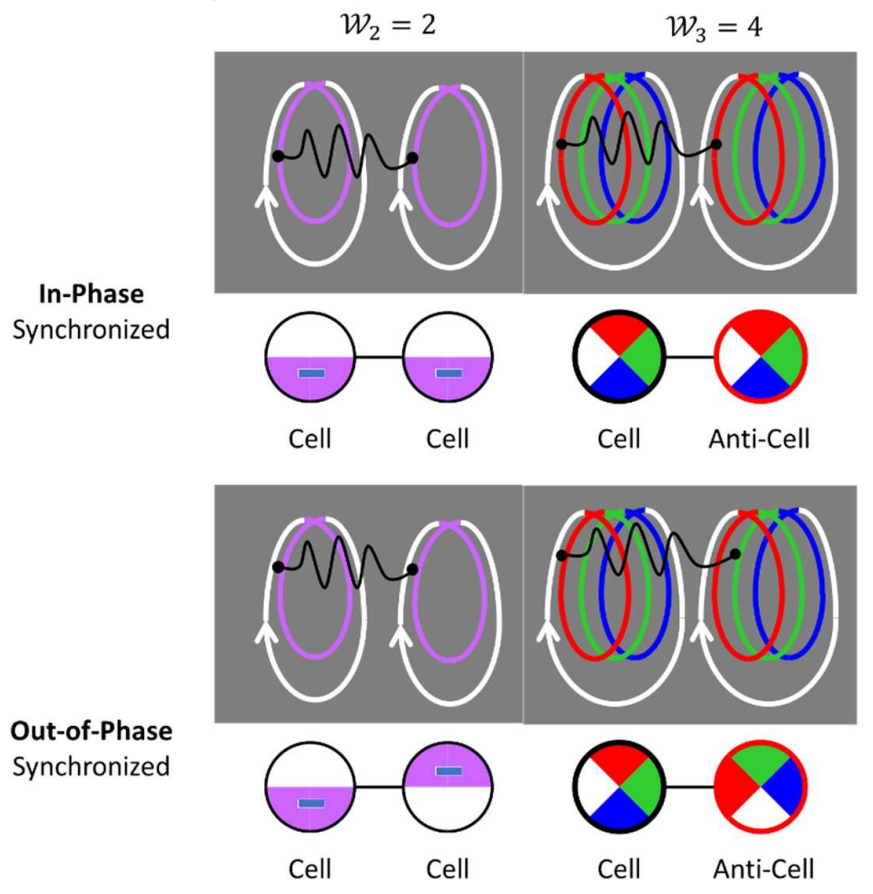

(a)

(b)

Figure 18. Spinorial cells represented by circular time diagrams (CTD): (a) in-phase synchronized; (b) outof-phase synchronized.

We assume that out-of-phase synchronized spinorial couples are stable, while in-phase synchronized couples are nonstable. Below is the explanation.

A quickly charge-flickering Phase 2 cell immersed into a Phase 1 (electromagnetic) network creates strong dynamic perturbations in the network. Regular cells surrounding the spinorial cell cannot follow these abrupt single-period charge changes. The situation is even more unstable, when two in-phase synchronized spinorial cells are immersed in the network. However, if the Phase 2 couple synchronizes out-of-phase (e.g. when one cell is charged and the other is neutral or vice versa), the amplitude of charge oscillation during a single period becomes much smaller. From the perspective of the surrounding cells, it appears as though a fixed-value charge is moving back and forth in space at very short distances. In comparison with perturbations imposed by a single spinorial cell, this perturbation is mild and does not destroy the network. The network responds to these types of perturbations by inducing currents which circulate around the couple and the corresponding magnetic moments. The apparent charge "motion" is not bound by the speed of light. We associate out-of-phase synchronized Phase 2 couples with first-generation leptons.

For a Phase 3 couple, the only way to achieve an apparently steady-state charge, is when the couple consists of one cell and one anti-cell. In time averaging, such a couple is neutral. Now, the only way to escape the couple's self-annihilation is for the cell and anti-cell to be synchronized out- 
of-phase. We associate out-of-phase synchronized Phase 3 cell-anti-cell couples with quarks. Quarks are electrically neutral and carry only color charges and flavors.

A benefit of this assignment is that we avoid the problem of fractional electric charges, which are empirically unobservable.

Correspondingly, hadrons (which can carry electrical charges) should consist of both quarks and leptons. In the proposed model, leptons carry electric charges. They also have up/down flavors. Quarks carry $(u / d \quad c / s \quad t / b)$ combined flavors (i.e. $u$-flavor and $d$-flavor are the same) and no electric charges. Combined quark flavors are associated with different synchronization patterns of their components. Flavor assignments for leptons and quarks are shown in Figure 19 with use of CTDs. Flavor $x / y$, corresponding to the in-phase synchronized quark, is unstable and only accounted for as a virtual state.

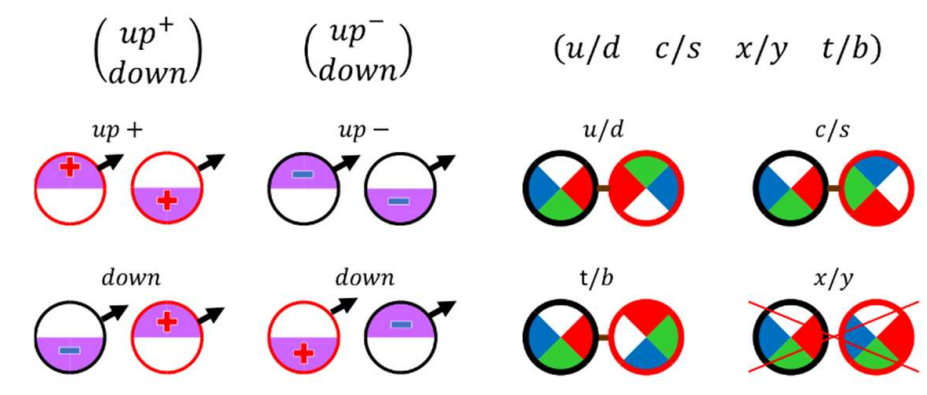

(a)

(b)

Figure 19. Flavor assignments: (a) Leptons; (b) Quarks.

The SM quark flavors can be recovered as a direct (Cartesian) product of quark $\left(\begin{array}{lll}u / d & c / s & t / b\end{array}\right)$-flavors and lepton $\left(\begin{array}{c}u p^{ \pm} \\ \text {down }\end{array}\right)$-flavors:

$$
\left(\begin{array}{ccc}
u & c & t \\
d & s & b
\end{array}\right)_{S M}=\left(\begin{array}{lll}
u / d & c / s & t / b
\end{array}\right) \times\left(\begin{array}{c}
u p^{ \pm} \\
\text {down }
\end{array}\right)
$$

Synchronized couples are bound states. The links between the cells are characterized by restoring forces $f_{j}(\psi)$ and energies $\varepsilon_{j}(\psi)$, and play the role of field mediators: photons (Phase 1), vector bosons (Phase 2), and gluons (Phase 3). In the former two cases, the links are elastic and the corresponding networks support elastic waves at small excitations.

In-phase synchronized links resemble solid-state massless acoustic phonons, while out-of-phase synchronized links resemble massive optical phonons. The Phase 1 network (electromagnetic) may carry only acoustic (massless) mediators, while stable spinorial clusters (particles) may carry only optical (massive) mediators.

Links between spinorial cells are inseparable from their host couples and are naturally localized inside the spinorial clusters (particles).

\section{Quark Flavor Changes}

Quarks and gluons of the proposed model are quite dynamic. The gluon charge-states follow the charge-states of their host cells. Here is an example of a charge cycle for one of the quark flavors: 


$$
\begin{gathered}
\text { Cell: } \ldots \rightarrow \text { neutral } \rightarrow \text { red } \rightarrow \text { green } \rightarrow \text { blue } \rightarrow \text { neutral } \rightarrow \cdots \\
\text { Anti }- \text { cell: } \ldots \rightarrow \text { red } \rightarrow \text { green } \rightarrow \text { blue } \rightarrow \text { neutral } \rightarrow \text { red } \rightarrow \cdots \\
\text { Gluon: } \ldots \rightarrow\left(\begin{array}{c}
\text { neutral } \\
\text { red }
\end{array}\right) \rightarrow\left(\begin{array}{c}
\text { red } \\
\text { green }
\end{array}\right) \rightarrow\left(\begin{array}{c}
\text { green } \\
\text { blue }
\end{array}\right) \rightarrow\left(\begin{array}{c}
\text { blue } \\
\text { neutral }
\end{array}\right) \rightarrow\left(\begin{array}{c}
\text { neutral } \\
\text { red }
\end{array}\right) \rightarrow \cdots
\end{gathered}
$$

When a quark flavor changes, its synchronization pattern also changes. Figure 20 shows all of the possibilities for one of the couple components changing its charge states while the other component remains intact. The direction of rotation is fixed and the same for cells and anti-cells. Each flavor-change step corresponds to CTD rotation across one-sector (90 degrees) on the cellular side (black circle on the left) or on the anti-cellular side (red circle on the right).

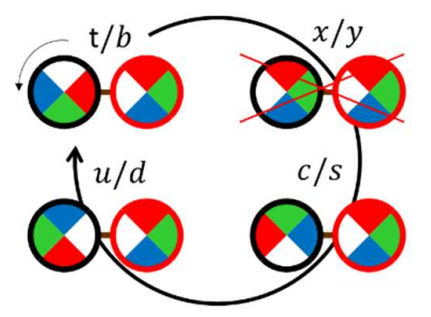

(a)

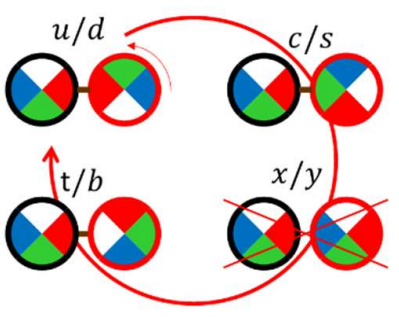

(b)

Figure 20. Quark flavor changes represented as CTD rotations: (a) on cell side; (b) on anti-cell side. Each flavor, with the exclusion of the unstable $x / y$, can be a starting point or an ending point.

Quark flavor changes can be associated with the following virtual process. A quark couple is briefly elevated to the level of dissociation. When the cells return, they have different synchronization patterns. The longer they stay at the elevated level, the bigger the changes in the synchronization patterns.

The probability of staying at the dissociation level exponentially decays with time

$$
w \approx \exp \left(-\varepsilon_{a} \Delta t\right),
$$

where $\varepsilon_{a}$ is the activation energy, and $\Delta t$ is the time spent at the elevated level. The activation energy (Figure 21) is the total internal energy of the couple, and consists of two cellular energies $2 \varepsilon_{\text {cell }} \sim 2 \mu_{3}$ and the synchronization energy, which according to (41), is equal to $\varepsilon_{\text {sync }} \sim 2 \varepsilon_{\text {cell }} \sim 2 \mu_{3}$. From (18), the chemical potential is $\mu_{3}=2 \mathcal{T}^{-1} \ln \delta_{F}$, and the activation energy is

$$
\varepsilon_{a}=8 \mathcal{T}^{-1} \ln \delta_{F} .
$$

Probability $w$ and probability amplitude $V$ are correspondingly

$$
w \approx \exp \left(-8 \mathcal{T}^{-1} \Delta t \ln \delta_{F}\right)=\delta_{F}^{-8 \mathcal{T}^{-1} \Delta t},
$$

and

$$
V=w^{1 / 2}=\delta_{F}^{-4 T^{-1} \Delta t}
$$




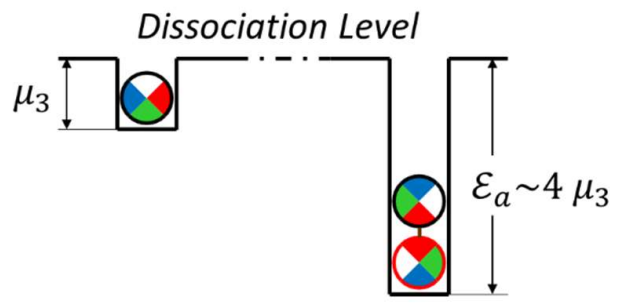

(a)

(b)

Figure 21. (a) Cellular chemical potential; (b) Quark activation energy

Below we estimate the probabilities $w$ and amplitudes $V$ of quark transmutations. For simplicity, we will only account for the shortest paths to get from one flavor to another, which correspond to the highest probabilities and amplitudes, while preserving unidirectional CTD rotations (due to time irreversibility). The shortest paths can be described as situations when one quark component (cell or anti-cell) continues to rotate at its original pace (in synchrony with other quarks and leptons in the cluster) while the other component is frozen. The temporal phase delay for the frozen component is $8 \pi \Delta t / \mathcal{T}$. Each $2 \pi$-phase delay implies a leap to the neighboring attractor loop (or one CTD sector rotation). Two examples are shown in Figure 22. The process resembles quantum tunneling through temporal barriers rather than spatial barriers.

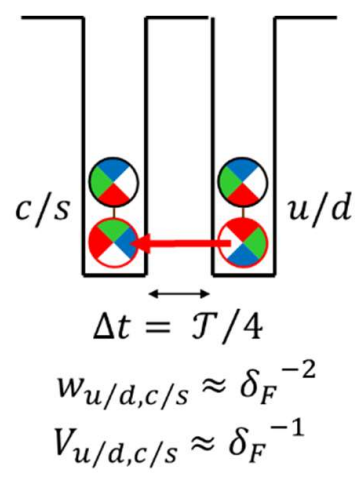

(a)

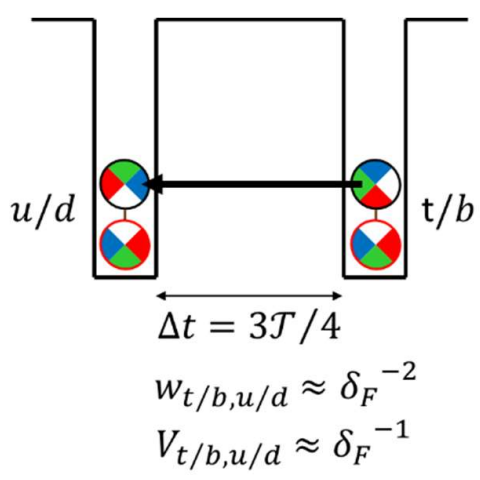

(b)

Figure 22. Examples of quark flavor change as temporal barrier tunneling: (a) through $1 / 4$ period barrier (from $u / d$ to $c / s$ ); (b) through $3 / 4$ period barrier (from $t / b$ to $u / d$ ). 
(a)

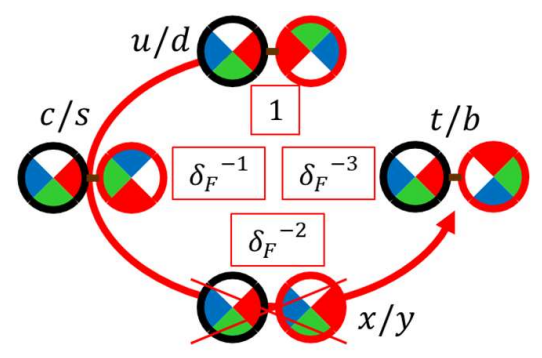

(c)

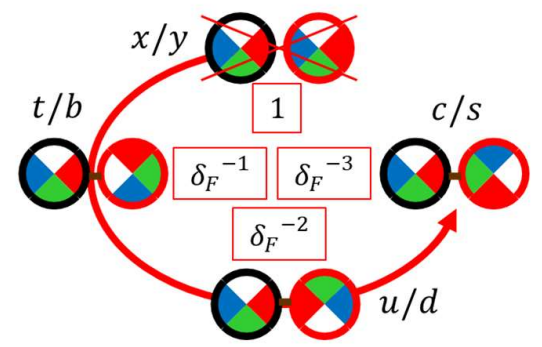

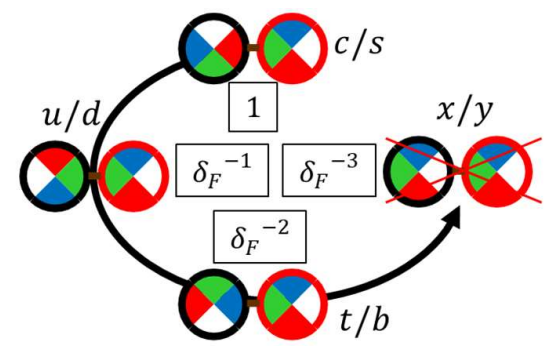

(b)

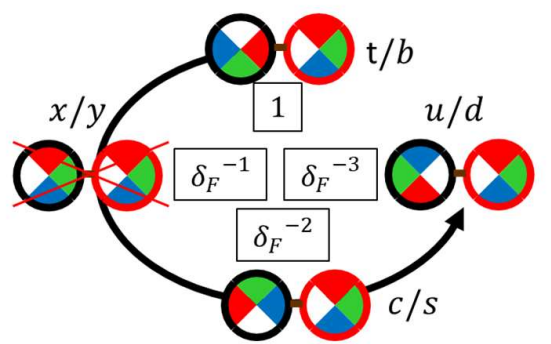

(d)

Figure 23. All possible quark flavor changes. Starting flavors are at the top of each diagram. The probability amplitudes $V$ are outlined by rectangles at the corresponding ending flavors. CTD rotations: (a) and (c) on anti-cell side; (b) and (d) on cell side.

Quark flavor rotations for all starting and ending flavors are shown in Figure 23. Starting flavors are at the top of each diagram. Anti-cell CTD rotation diagrams are on the left, while cell CTD rotation diagrams are on the right. Diagram in Figure 23(c) corresponds to an unstable starting flavor. The probability amplitudes calculated using (54) are outlined by rectangles at the corresponding ending flavors.

Quark flavor changes from Figure 23 are shown as a $4 \times 4$ matrix in Figure 24(a).

\begin{tabular}{|c|c|c|c|c|}
\cline { 2 - 4 } \multicolumn{1}{c|}{} & $u / d$ & $c / s$ & $x / y$ & $t / b$ \\
\hline$u / d$ & 1 & $\delta_{F}{ }^{-1}$ & $\delta_{F}{ }^{-2}$ & $\delta_{F}{ }^{-3}$ \\
\hline$c / s$ & $\delta_{F}^{-1}$ & 1 & $\delta_{F}^{-3}$ & $\delta_{F}^{-2}$ \\
\hline$x / y$ & $\delta_{F}^{-2}$ & $\delta_{F}^{-3}$ & 1 & $\delta_{F}^{-1}$ \\
\hline$t / b$ & $\delta_{F}^{-3}$ & $\delta_{F}^{-2}$ & $\delta_{F}^{-1}$ & 1 \\
\hline
\end{tabular}

(a)

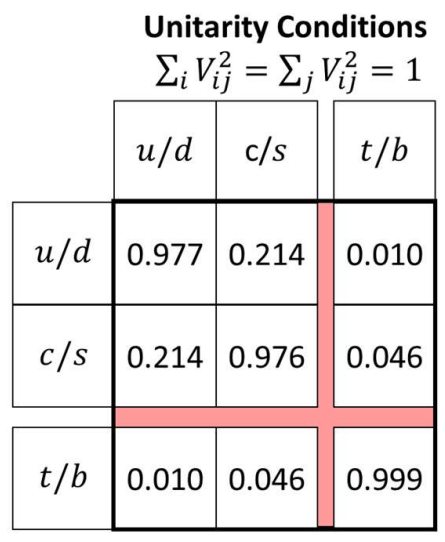

(b)
CKM-Matrix

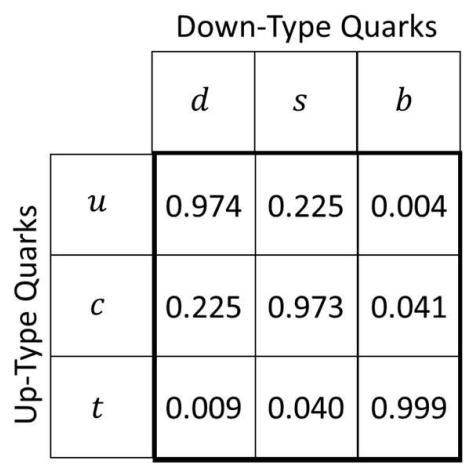

(c)

Figure 24. Quark flavor change probability amplitudes in matrix representations: (a) original $4 \times 4$ matrix; (b) $3 \times 3$ matrix without the unstable $x / y$ flavors; (c) Cabibbo-Kobayashi-Maskawa matrix. In (b) and (c), numbers are rounded. 
Row and column designations represent starting/ending flavors. Probability amplitudes are shown as matrix elements at cross-sections of the corresponding rows and columns. The matrix has appearance of the Klein four-group $V_{4}$ Cayley table. After removing the third row and third column (shown in pink in Figure 24(a)), which correspond to the unstable starting/ending flavor $x / y$, and reducing the main diagonal elements to satisfy row/column unitarity, we obtain a $3 \times 3$ matrix shown in Figure 24(b). For comparison, the Cabibbo-Kobayashi-Maskawa (CKM) matrix [15] with empirically obtained quark mixing amplitudes is shown in Figure 24 (c).

\section{Particles}

Despite having only two cells and two anti-cells in our particle building kit, their rich dynamics and diversity of synchronization patterns allows us to synthesize the entire family of "elementary" particles, which resemble those described by the Standard Model. A few examples are shown in Figures 25-27. The particles are represented by CTDs. Synchronization patterns are different for different particles. All links represent out-of-phase synchronizations.

Leptons are built from Phase 2 cells/anti-cells (Figure 25). First-generation leptons possess two cells (electron), two anti-cells (positron), or combination cell and anti-cell (neutrino) Figure 25(a). Second-generation leptons (Figure 25(b)) also have one neutral couple (cell and anti-cell), and thirdgeneration leptons (Figure 25(c)), have two neutral couples. Adding neutral couples does not affect the electric charge pattern, but does increase the particle internal energies (masses).
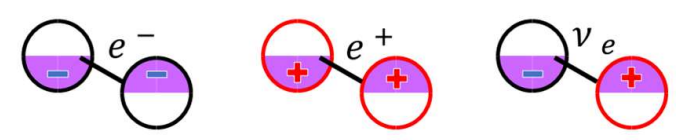

(a)
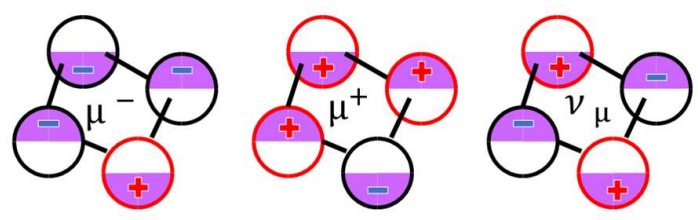

(b)
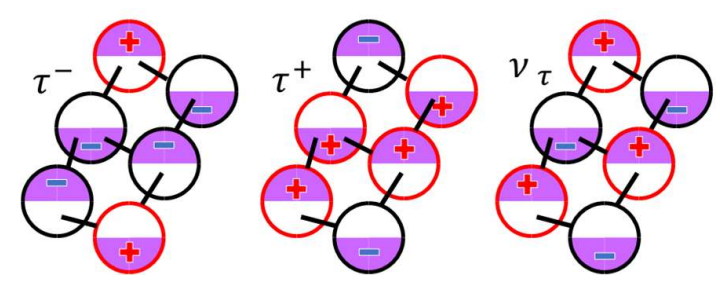

(c)

Figure 25. Lepton family: (a) first generation; (b) second generation; (c) third generation

Mesons are synthesized by replacing one neutral Phase 2 couple with one neutral Phase 3 couple (quark) in second-generation leptons. A meson octet resembling the Eightfold Way group of real (SM) mesons [9] is shown in Figure 26. Due to the significantly richer dynamics of Phase 3 cells, this replacement essentially increases the number of different particles. 


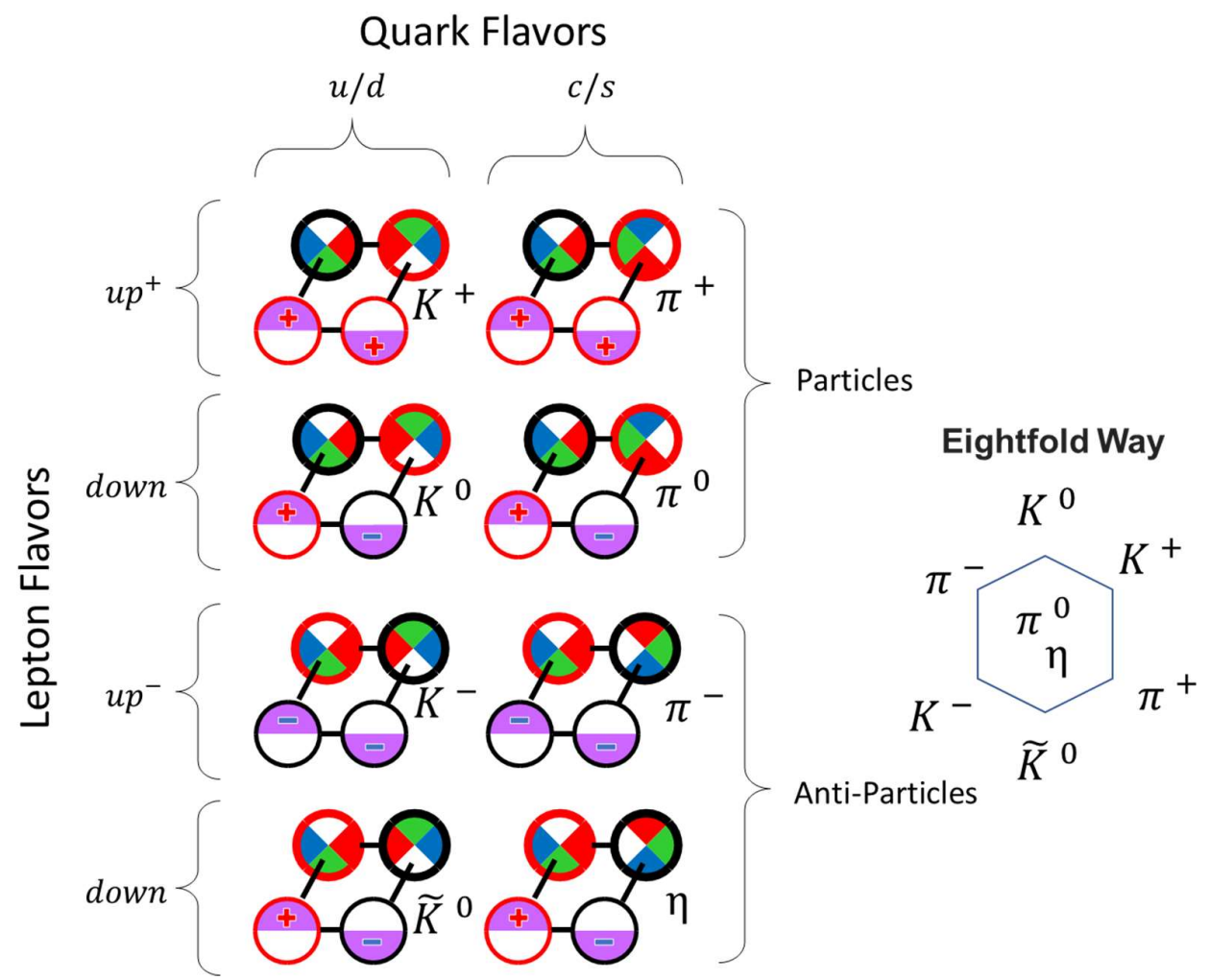

Figure 26. "Eightfold Way" meson octet

Baryons are synthesized by replacing two neutral Phase 2 couples with two quarks in thirdgeneration leptons. The number of particles again increases. A baryon octet resembling the Eightfold Way group of real baryons [9] is shown in Figure 27.

Quark flavors

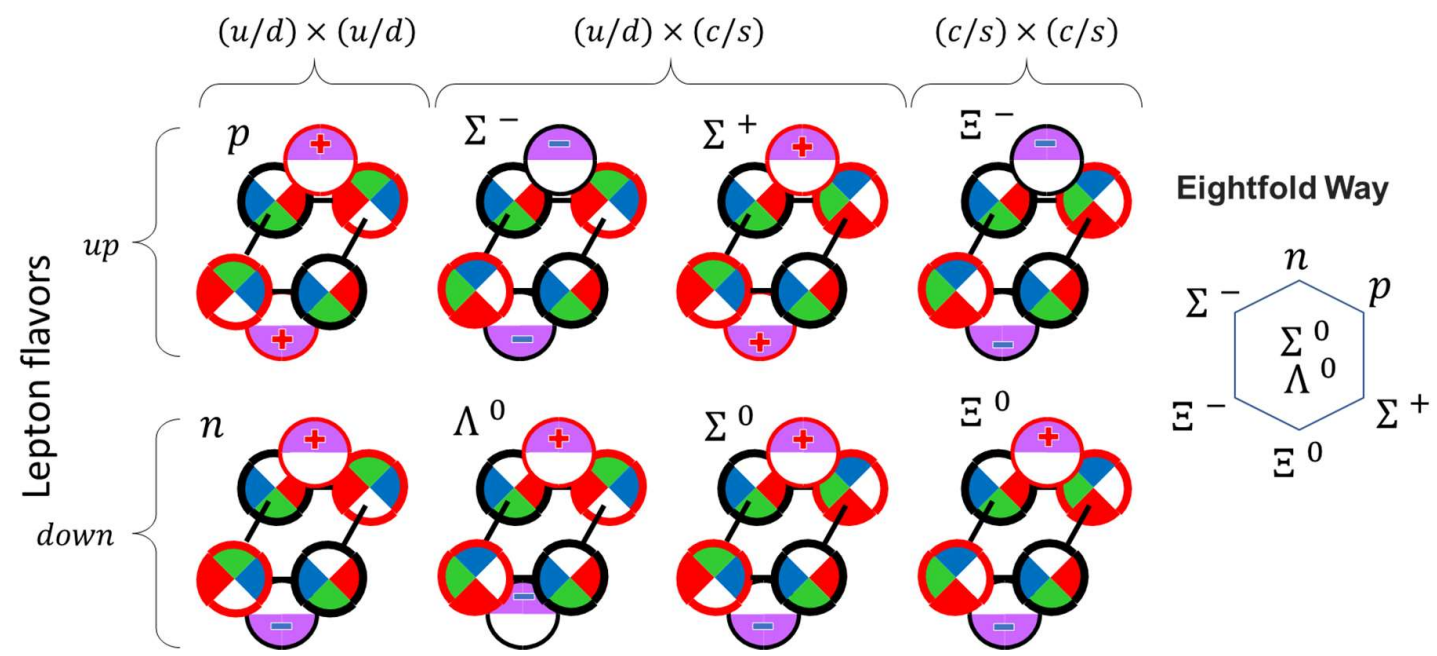

Figure 27. "Eightfold Way" baryon octet 
CTDs may also illustrate nuclear reactions, as the example shown in Figure 29.

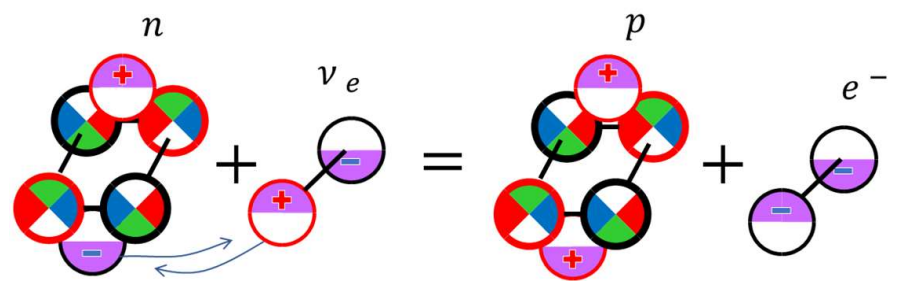

Figure 29. Reaction $n \rightarrow p+e^{-}+\tilde{v}_{e}$ represented by cellular circular time diagrams. The anti-neutrino in the right portion of the equation is replaced by the neutrino in the left portion.

When a number of vacuum cells synchronize, they form a coherent network. Phase differences $\psi$, restoring forces $f$ and energies $\mathcal{E}$ become tensors parameterized by their links. An example of a cell surrounded by six other cells of a different nature and with different synchronization patterns is shown in Figure 30(a). The same cellular cluster half-period later is shown in Figure 30(b).

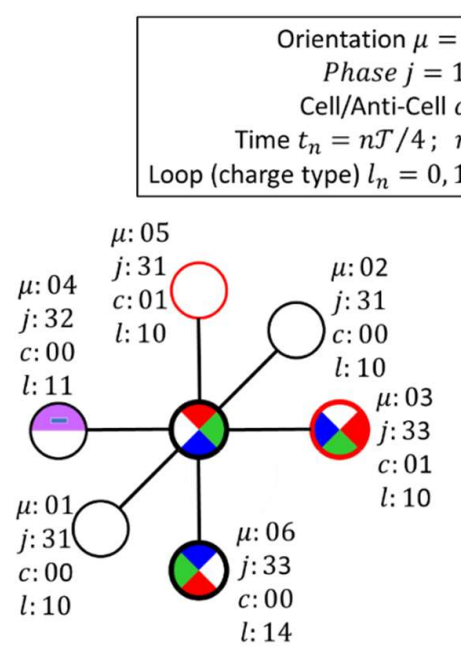

(a)
Phase $j=1,2,3$ Lop (charge type) $l_{n}=0,1,2,3 ; l \leq 2^{j-1}-1$

(b)

Figure 30. (a) Link parametrization for a cellular cluster comprising a Phase 3 cell (diagram centers) surrounded by six other cells of a different nature and with different synchronization patterns; (b) the same cluster half-period later.

The six links are parameterized by their directions $(\mu=01,02,03, \ldots 06)$, cell types $(c=0$ for cells, $c=1$ for anti-cells), Phases $j(j=1,2,3)$, and attractor loop numbers $(l=0,1,2,3)$. Parameters $\mu, c, j$, and $l$ are different for different depending on the types of the adjacent cells and their synchronization patterns. For links comprising at least one Phase 2 cell but no Phase 3 cells, parameter $l$ changes each half-period. For links comprising at least one Phase 3 cell, parameter $l$ changes each quarter-period.

From a bird's eye view, vacuum cellular networks look like continuous media. The natural way to describe their dynamics would be to use fiber bundle construction on space-time manifold where the orientation $\mu$ is replaced by spatial and temporal coordinates, and the intracellular and intercellular dynamics are elevated to the fiber spaces. 


\section{Conclusion}

We have described a potential scenario for the emergence of coherent, dynamically rich cellular networks in a gravitation-diffusion driven vacuum. The network patterns belonging to different phases emulate electromagnetic, weak, and strong nuclear fields, while spinorial cell inclusions in the electromagnetic network imitate leptons and quarks.

The proposed model provides an explanation for the origin of various quantum phenomena that have not been solved by current theory. This model is causal, strictly obeying time irreversibility. It describes global symmetries (like universal time scale, "relativistic" time dilation, spatial quantum coherency, quantum statistics) as phenomena that emerge with and are inseparable from cellular vacuum networks as their carriers.

The model also allows for rough estimations of relative field strengths, quark transmutation amplitudes, the quantity of field charges, and quark flavors in fair agreement with experimental data.

The proposed model belongs to the Feigenbaum universality and is based on the ubiquitous phenomenon of synchronization. Therefore, the main results and numerical estimations are generalizable and not sensitive to the details of the model such as the selection of an explicit form of an iteration function.

Funding: This research received no external funding.

Acknowledgments: I would like to express my special gratitude to my children, Julia Manasson and Alexander Manasson, for reading the manuscript and making useful suggestions.

Conflicts of Interest: The author declares no conflict of interest.

\section{References}

1. Dynamics of Spatio-Temporal Cellular Structures: Henri Bénard Centenary Review; Mutabazi, I.; Wesfreid, J.E.; Guyon, E., Eds. Springer Tracts in Modern Physics 207; Springer-Verlag: New York, 2006; 249 pp., ISBN 0-387-40098-2.

2. Peitgen, H.O.; Jürgens, H.; Saupe, D. Chaos and Fractals: New Frontiers of Science; Springer-Verlag: New York, 1992; 984 pp., ISBN 0-387-97903-4.

3. The Logistic Map and the Route to Chaos: From the Beginnings to Modern Applications; Ausloos, M.; Dirickx, M., Eds.; Springer: Berlin, 2006; 411 pp., ISBN 3-540-28366-8

4. Hilborn, R.C. Chaos and Nonlinear Dynamics: An Introduction for Scientists and Engineers; Oxford University Press: New York, 1994; 654 pp., ISBN 0-19-505760-0.

5. Feigenbaum, M.J. Quantitative Universality for a Class of Non-Linear Transformations. J. Stat. Phys. 1978, 19, 25-52. https://doi.org/10.1007/BF01020332

6. Feigenbaum, M.J. Universal Metric Properties of Non-Linear Transformations. J. Stat. Phys. 1979, 21, 669-706. https://doi.org/10.1007/BF01107909

7. Cvitanović, P. Universality in Chaos. Acta Physica Polonica 1984, A 65, 203-239.

8. Physical Constants; Reviewed 2015 by Mohr, P.J.; Newell, D.B. http://pdg.lbl.gov/2018/reviews/rpp2018-rev-phys-constants.pdf (accessed on 03/10/2018).

9. Griffiths, D.J. Introduction to Elementary Particles; John Willey \& Sons: New York, 1987; 392 pp., ISBN 0471-60386-4.

10. Pikovsky, A.; Rosenblum, M.; Kurths, J. Synchronization: A Universal Concept in Nonlinear Sciences; Cambridge University Press: Cambridge, 2003; 411 pp., ISBN 0-521-59285-2.

11. Strogatz, S.H. From Kuramoto to Crawford: Exploring the Onset of Synchronization in Populations of Coupled Oscillators. Physica 2000, 143D, 1-20.

12. Adler, R. A Study of Locking Phenomena in Oscillators. Proc. IRE 1946, 34, 351-357; Reprinted in Proc. IEEE 1973, 61, 1380-1385. https://doi.org/10.1109/PROC.1973.9292

13. Mirollo, R.E.; Strogatz, S. H. Amplitude Death in an Array of Limit-Cycle Oscillators. J. Stat. Phys. 1990, 60, 245-262. https://doi.org/10.1007/BF01013676 
14. Koseska, A.; Volkov, E.; Kurths, J. Oscillation Quenching Mechanisms: Amplitude vs Oscillation Death. Phys. Rep. 2013, 531, 173-199. https://doi.org/10.1016/j.physrep.2013.06.001

15. CKM Quark-Mixing Matrix. Revised (January 2018) by Ceccucci, A.; Ligeti, Z.; Sakai, Y.; http://pdg.lbl.gov/2018/reviews/rpp2018-rev-ckm-matrix.pdf (accessed on 03/10/2018). 